\title{
Interfacial dislocation motion and interactions in single-crystal superalloys
}

\author{
B. Liu ${ }^{a, b, *}$, D. Raabe ${ }^{b}, F \cdot$ Roters $^{b}, A \cdot$ Arsenlis $^{a}$

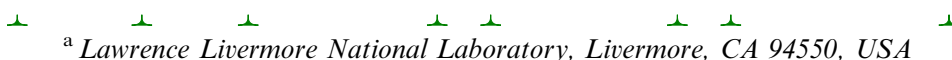 \\ b Max-Planck-Institut für Eisenforschung, 40237 Düsseldorf, Germany
}

Received 4 February 2014; received in revised form 18 June 2014; accepted 19 June 2014

\begin{abstract}
The early stage of high-temperature low-stress creep in single-crystal superalloys is characterized by the rapid development of interfacial dislocation networks. Although interfacial motion and dynamic recovery of these dislocation networks have long been expected to control the subsequent creep behavior, direct observation and hence in-depth understanding of such processes has not been achieved. Incorporating recent developments of discrete dislocation dynamics models, we simulate interfacial dislocation motion in the channel structures of single-crystal superalloys, and investigate how interfacial dislocation motion and dynamic recovery are affected by interfacial dislocation interactions and lattice misfit. Different types of dislocation interactions are considered: self, collinear, coplanar, Lomer junction, glissile junction and Hirth junction. The simulation results show that strong dynamic recovery occurs due to the shortrange reactions of collinear annihilation and Lomer junction formation. The misfit stress is found to induce and accelerate dynamic recovery of interfacial dislocation networks involving self-interaction and Hirth junction formation, but slow down the steady interfacial motion of coplanar and glissile junction forming dislocation networks. The insights gained from these simulations on high-temperature low-stress creep of single-crystal superalloys are also discussed.
\end{abstract}

(C) 2014 Published by Elsevier Ltd. on behalf of Acta Materialia Inc.

Keywords: Dislocation dynamics; Superalloys; High-temperature low-stress creep; Interfacial dislocation motion; Dislocation interactions

\section{Introduction}

Single-crystal superalloys are used as turbine blade materials because of their excellent creep resistance during the operation of gas turbines in aircraft and power-generation engines. The microstructure of these alloys consists of $\gamma$ matrix (face-centered cubic) containing a high volume fraction of cuboidal $\gamma^{\prime}$ particles $\left(L 1_{2}\right.$ lattice). Creep deformation in these structures exhibits three regimes: a low-temperature and high-stress regime (e.g. $750{ }^{\circ} \mathrm{C}, 750 \mathrm{MPa}$ ), where plastic strain is accumulated by $\langle 112\rangle$ dislocation ribbons cutting the $\gamma^{\prime}$ precipitates $[1,2]$; an intermediate-temperature and stress regime (e.g. $900{ }^{\circ} \mathrm{C}, 450 \mathrm{MPa}$ ), where plastic

* Corresponding author at: Lawrence Livermore National Laboratory, Livermore, CA 94550, USA.

E-mail address: bingliu@1lnl.gov (B. Liu). deformation occurs by Orowan bypassing of $1 / 2\langle 011\rangle$ dislocations through the $\gamma$ matrix channels $[3,4]$; and a high-temperature and low-stress regime (e.g. $1100{ }^{\circ} \mathrm{C}$, $137 \mathrm{MPa}$ ), where plastic deformation initializes easily as the grown-in $1 / 2\langle 011\rangle$ dislocations glide in the horizontal channels [5-7], proceeds slowly by the motion of $1 / 2$ $\langle 011\rangle$ dislocations along the interfaces [8-10], and eventually accelerates via $\langle 100\rangle$ superdislocations cutting the $\gamma^{\prime}$ precipitates $[11,12]$.

Interfacial dislocation motion and interactions occur in the high-temperature and low-stress regime. These interfacial dislocations are generated by the glide of grown-in dislocations driven jointly by the applied stress and misfit stress in the horizontal channels [13-15]. As more dislocations are deposited on the interface, the internal stresses associated with the interfacial dislocation networks soon become high enough to prevent the further propagation 
of the grown-in dislocations, and thus end primary creep $[5,16,17]$. Interfacial dislocation motion, by a combination of glide and climb, becomes the main deformation process during secondary creep that constitutes the majority of the superalloy's creep life [9,10,18,19].

The objective of this work is to investigate how interfacial dislocation motion in the $\gamma / \gamma^{\prime}$ structure is affected by longrange and short-range dislocation interactions in interfacial dislocation networks using discrete dislocation dynamics (DDD) simulations. Three-dimensional DDD models [2025] are robust tools to study elementary dislocation interactions [26-28], and the strengthening effect of dislocation interactions in nanoscale metallic multilayered composites [29], microscale plasticity [30-32] and coarse-grained crystals at low-angle grain boundaries $[33,34]$. The present work is focused on the role of interfacial dislocation interactions during high-temperature creep of single-crystal superalloys. In previous studies [26-29,31-34], dislocation interactions were generally found to contribute to material strength and cause strain hardening. In this work, we show that interfacial dislocation interactions may also induce dynamic recovery, which relieves internal stress and leads to creep softening. Although dynamic recovery has long been assumed to occur during secondary creep of single-crystal superalloys $[5,9,10,18,19]$, direct observation and hence indepth understanding of such processes has not been reached. In the $\gamma$ phase matrix channels, positive and negative dislocations are forced by the external stresses onto opposite interfaces, so that dynamic recovery does not proceed as in pure metals where dislocations of opposite signs directly meet and annihilate. Our simulations of interfacial dislocation motion and interactions will cast light on the recovery mechanisms during high-temperature low-stress creep in these channel structures.

We will first introduce our model to simulate interfacial dislocation motion and interactions in the $\gamma / \gamma^{\prime}$ structure, which includes model modifications that enable dislocation climb driven by mechanical and chemical forces, incorporates the antiphase boundary back-driving force in the precipitates, and considers the biaxial misfit stresses in the matrix channels. The simulations of interfacial dislocation motion during creep are then presented, which consider different initial configurations, namely mixed dislocations, edge dislocations and dislocation networks involving different types of dislocation interactions (self, collinear, coplanar, Lomer junction, glissile junction and Hirth junction). In each case, the role of the misfit stress is also investigated by running two separate simulations with the misfit stress in the model being switched on and off, respectively. The insights gained from these simulations on high-temperature low-stress creep in single-crystal superalloys will be finally discussed and summarized.

\section{Simulation method}

Dislocations climb through emission and adsorption of vacancies at jogs. Under the condition of high temperature and low stress, the jog density is high enough that each point along the dislocation line may act as a source or sink of vacancies, and the vacancy concentration is approximately uniform along the dislocation core [35]. The velocity of dislocation climb is then controlled by vacancy diffusion [35-42]:

$V_{c}=\frac{2 \pi \Omega D_{v} c_{0} F_{c}}{b^{2} \ln (R / b) k_{B} T}$,

where $D_{v}$ is the vacancy diffusion coefficient, $c_{0}$ is the equilibrium vacancy concentration, $F_{c}$ is the climb force, $\Omega$ is the atomic volume, $b$ is the magnitude of the Burgers vector, $R$ is a distance from the dislocation core over which the vacancy concentration reaches its average value in the sample, $k_{B}$ is Boltzmann's constant, and $T$ is the absolute temperature.

A drag relation between the climb force and velocity can be derived from Eq. (1):

$V_{c}=\frac{F_{c}}{B_{c}}, \quad B_{c}=\frac{b^{2} \ln (R / b) k_{B} T}{2 \pi \Omega D_{v} c_{0}}$,

where the drag coefficient $B_{c}$ is a function of the vacancy diffusion coefficient $D_{v}$, the equilibrium vacancy concentration $c_{0}$, and the temperature $T$. It is worth mentioning that a similar climb force-velocity drag relation has also been derived in the case of low jog density (source/sink-controlled climb) [43].

The climb motion of dislocations can be described in a drag-type relation similar to the glide motion, which allows dislocation dynamics models to handle dislocation glide and climb in the same framework [25,39,41,43-45].

We use the ParaDiS DDD code [25], which defines the nodal force and velocity in a drag-type relation:

$\boldsymbol{F}_{i}=\frac{1}{2} \sum_{j}\left\|\boldsymbol{l}_{i j}\right\| \boldsymbol{B}_{i j} \boldsymbol{V}_{i}$

where $\boldsymbol{F}_{i}$ is the force on node $i, j$ is a node connected to $i$ through a line segment $\boldsymbol{l}_{i j}, \boldsymbol{B}_{i j}$ is the drag tensor (inverted mobility tensor) for segment $\boldsymbol{l}_{i j}$, and $\boldsymbol{V}_{i}$ is the calculated nodal velocity. The drag tensor $\boldsymbol{B}$ enforces spatial constraints on the dislocation motion in face-centered cubic (fcc) crystals according to:

$$
\boldsymbol{B}=\left\{\begin{array}{cc}
B_{g}(\boldsymbol{m} \otimes \boldsymbol{m})+B_{c}(\boldsymbol{n} \otimes \boldsymbol{n})+B_{l}(\boldsymbol{t} \otimes \boldsymbol{t}) & \boldsymbol{n} \| 111 \\
B_{c} \boldsymbol{I}+\left(B_{l}-B_{c}\right)(\boldsymbol{t} \otimes \boldsymbol{t}) & \boldsymbol{n} \nmid 111
\end{array}\right.
$$

where the drag coefficient $B_{g}$ controls dislocation glide on the glide plane and perpendicular to the dislocation line, $B_{c}$ defines dislocation climb along the glide plane normal $\boldsymbol{n}$, and $B_{l}$ is the drag coefficient associated with moving a node along its line direction $\boldsymbol{t}$.

The fcc mobility law in the ParaDiS code $[25,46]$, defined through Eqs. (3) and (4), was designed primarily for dislocation glide on $\{111\}$ planes, and $B_{c}$ was set large enough to prevent glide on non- $\{111\}$ planes and climb in general. As dislocations lose their initial glide planes in a threedimensional DDD simulation incorporating dislocation 
climb, e.g. activation of a Bardeen-Herring source [38], glide on $\{111\}$ and non- $\{111\}$ planes should be treated in a similar fashion [39]. In this work, we thus modified the non$\{111\}$ drag tensor to be the same as the $\{111\}$ drag tensor:

$\boldsymbol{B}=B_{g}(\boldsymbol{m} \otimes \boldsymbol{m})+B_{c}(\boldsymbol{n} \otimes \boldsymbol{n})+B_{l}(\boldsymbol{t} \otimes \boldsymbol{t})$,

$$
\boldsymbol{n} \| 111 \cup \boldsymbol{n} \nmid 111 \text {. }
$$

The nodal force in the model is calculated from the forces exerted on the segments that the node connects:

$\boldsymbol{F}_{i}=\sum_{j} \boldsymbol{f}_{i j}$.

The segment force $\boldsymbol{f}_{i j}$ has multiple sources:

$\boldsymbol{f}_{i j}=\boldsymbol{f}_{i j}^{\text {core }}+\boldsymbol{f}_{i j}^{\text {elastic }}+\boldsymbol{f}_{i j}^{\text {external }}+\boldsymbol{f}_{i j}^{\text {osmotic }}+\boldsymbol{f}_{i j}^{A P B}+\boldsymbol{f}_{i j}^{\text {misfit }}$.

$\boldsymbol{f}_{i j}^{c o r e}$ is the line tension force that minimizes the dislocation core energy by reducing the segment length and rotating the segment towards lower-energy orientations. $\boldsymbol{f}_{i j}^{\text {elastic }}$ is the elastic force due to the stress field of the dislocation network. $\boldsymbol{f}_{i j}^{\text {external }}$ is the external force due to the remotely applied stress. $\boldsymbol{f}_{i j}^{\text {osmotic }}$ is the osmotic force due to vacancy depletion or supersaturation. $\boldsymbol{f}_{i j}^{A P B}$ is the antiphase boundary (APB) back-driving force to prevent dislocation cutting of the $\gamma^{\prime}$ precipitates. $\boldsymbol{f}_{i j}^{\text {misfit }}$ is the force due to the presence of misfit stress in the $\gamma / \gamma^{\prime}$ structures. The explicit forms of $\boldsymbol{f}_{i j}^{\text {core }}, \boldsymbol{f}_{i j}^{\text {elastic }}$, and $\boldsymbol{f}_{i j}^{\text {external }}$ are given in Ref. [25]. The expressions for $\boldsymbol{f}_{i j}^{\text {osmotic }}, \boldsymbol{f}_{i j}^{A P B}$, and $\boldsymbol{f}_{i j}^{\text {misfit }}$ are listed below.

The osmotic force is in the following form $[38,39,46,47]$ :

$\boldsymbol{f}_{i j}^{o s m o t i c}=-\frac{k_{B} T}{2 \Omega} \ln \left(\frac{c_{\infty}}{c_{0}}\right) \boldsymbol{b}_{i j} \times \boldsymbol{l}_{i j}$,

where $\boldsymbol{b}_{i j}$ is the Burgers vector of the dislocation segment, and $c_{\infty}$ is the average vacancy concentration in the sample.

The APB back-driving force is implemented as:

$\boldsymbol{f}_{i j}^{A P B}=\left\{\begin{array}{cc}\frac{1}{2} \chi^{A P B}\left\|\boldsymbol{l}_{i j}\right\| \sin \left(\frac{\pi}{2} \cdot \frac{L-d}{L}\right) \boldsymbol{n}^{s} & d \leqslant L \\ 0 & d>L\end{array}\right.$

where $\chi^{A P B}$ is the APB energy per unit area, $d$ is the minimum distance from the segment center to the precipitate surface, $L$ is a transition length over which the APB back-driving force per unit length decays from $\chi^{A P B}$ to zero, and $\boldsymbol{n}^{s}$ is the outward normal of the cuboidal precipitate surface pointing to the segment center. For the calculations of the minimum distance $d$ and the outward surface normal $\boldsymbol{n}^{s}$, the surface areas at cube edges and corners are approximated as one-quarter of a cylinder and one-eighth of a sphere, respectively, whose radii are both set to be the same as the transition length $L$. Similar APB back-driving force models have been applied in the DDD simulations of dislocation-precipitate interactions at low and intermediate temperatures, where only dislocation glide is considered, and the direction of the APB back-driving force can be simply set to the opposite direction of dislocation glide [48-52]. In this work, we consider both dislocation glide and climb, and the back-driving force direction is aligned with the outward-pointing surface normal, which efficiently prevents dislocations from entering the precipitates, but allows tangential dislocation motion along the interface.

The force exerted by the misfit stress is calculated using the Peach-Koehler equation:

$\boldsymbol{f}_{i j}^{\text {misfit }}=\frac{1}{2} \boldsymbol{\sigma}^{m i s f i t} \cdot \boldsymbol{b}_{i j} \times \boldsymbol{l}_{i j}$.

The misfit stress is caused by the lattice mismatch between the $\gamma$ and $\gamma^{\prime}$ phases in the nickel-based superalloys, which has been extensively investigated in the past using finite-element (FE) calculations [3-5,7,53-57]. These FE calculation results show that the negative lattice misfit produces biaxial compressive stresses in the matrix, which are parallel to the interfaces and rather uniform in each of the matrix channels. Accordingly, the following misfit stress tensors $\boldsymbol{\sigma}^{\text {misfit }}$ are considered in the model for the matrix channels normal to $X, Y$ and $Z$ directions, respectively:

$$
\begin{aligned}
& \text { channels }(\perp X) \quad \text { channels }(\perp Y) \quad \text { channels }(\perp Z)
\end{aligned}
$$

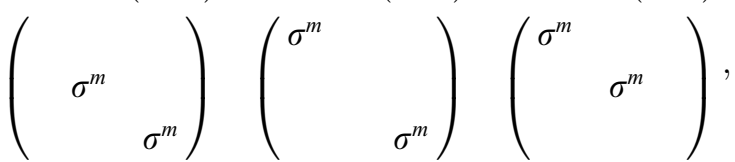

where $\sigma^{m}$ denotes the misfit stress components. A similar implementation of the misfit stress has been previously used in the DDD simulations of Huang et al. [52].

\section{Results}

The interfacial dislocation motion and interactions are simulated by applying a tensile stress of $137 \mathrm{MPa}$ along the [0 01 1] direction. For simplicity, isotropic elasticity is adopted with a shear modulus $\mu$ of $44.22 \mathrm{GPa}$ and a Poisson ratio $v$ of 0.37 . The dislocation glide drag coefficient $B_{g}$ is set to be $1 \%$ of the dislocation climb drag coefficient $B_{c}, B_{g}=\frac{1}{100} B_{c}$, to reflect that glide is substantially faster than climb. Similar to the level set dislocation dynamics simulations of Quek et al. [45], the simulation time in this work is normalized by $B_{c} / \mu$, the quotient of the dislocation climb drag coefficient and the shear modulus, so that the actual value of dislocation climb drag coefficient is not required for the simulation. In the simulated $\gamma / \gamma^{\prime}$ microstructure, the edge length of the cuboidal $\gamma^{\prime}$ particles is $1600 \mathrm{~b}$, and the width of the $\gamma$ channels is $400 \mathrm{~b}$. The magnitude of the Burgers vector $b$ is $0.25 \mathrm{~nm}$. The APB energy $\chi^{A P B}$ in the $\gamma^{\prime}$ precipitates is set to be $125 \mathrm{~m} \mathrm{~J} \mathrm{~m}^{-2}$ based on the ab initio simulation result of Yashiro et al. [50]. The misfit stress component $\sigma^{m}$ in the matrix $\gamma$ channels is set to be $-100 \mathrm{MPa}$ based on the FE calculations by Zhang et al. [7]. The vacancies generated by dislocation climb under the tensile stress are largely adsorbed through porosity growth in the superalloys [10]. The average vacancy concentration in the sample is assumed to be at the equilibrium value, i.e. $c_{\infty}=c_{0}$, and the osmotic force defined in Eq. (8) is thus zero in all the simulations. The effect of vacancy supersaturation will be investigated in future work.

The simulation results can be generally divided into two groups according to the initial configurations: widely 
separated interfacial dislocations with the same line sense on the same side of the horizontal channels; and closely spaced interfacial dislocations with opposite line senses on opposite sides of the horizontal channels. The interfacial dislocations are initially all on the horizontal interfaces based on the fact that the superposition of external and misfit stresses favors the slip deposition in the horizontal channels. The simulation results for different initial configurations are presented by two means: simulation snapshots showing the actual sequence of dislocation motion and interactions, and curve diagrams presenting the corresponding plastic strain accumulation and dislocation density evolution with time.

\subsection{Interfacial motion of widely separated dislocations}

The simulations with widely separated dislocations are designed to investigate interfacial dislocation motion in the absence of considerable internal stresses, where dislocation movement is dominated by the external stress, misfit stress and line tension of the dislocation. 1/2 [0 011 l] Interfacial dislocations of mixed and edge characters are considered, and their initial configurations are shown in Fig. 1. Mixed interfacial dislocations are generated by the glide of grown-in $1 / 2\langle 011\rangle\{11 \overline{1}\}$ dislocations in the horizontal channels, and their line direction is along the intersection line of the glide plane and the $\gamma / \gamma^{\prime}$ interface normal to the $[001]$ direction. Edge interfacial dislocations are formed by interfacial dislocation reactions as will be discussed later. Such edge dislocations are also the basic dislocation configuration considered in various models to interpret high-temperature low-stress creep in single-crystal superalloys $[10,18,19,58]$.

Fig. 2 shows the plastic strain accumulation and dislocation density evolution with time in these simulations, which are clearly affected by both the character of the interfacial dislocations and the presence of the misfit stresses.

\subsubsection{Mixed dislocations}

The simulation of mixed dislocations without considering the misfit stresses is shown in Fig. 3(a). The dislocations initially move along the horizontal interfaces and partially enter the vertical channels (Fig. 3(a), image 1), then become fully trapped in the vertical channels and start to move upwards along the vertical interfaces (Fig. 3(a), image 2), and eventually re-enter the horizontal channels and move again along the horizontal interfaces (Fig. 3(a), image 3). The following snapshots of this simulation record the repetition of these processes, and are thus not shown in the paper. While the dislocations can enter and cross the $\gamma$ matrix channels simply by glide, the dislocation motion along the $\gamma / \gamma^{\prime}$ interfaces can only be achieved by a combination of glide and climb.

The applied normal stress along the [0 010 direction generates a Peach-Koehler force in the (001) plane, which activates the dislocation motion along the horizontal interfaces (normal to the [0 01 1] direction), but does not affect the dislocation motion along the vertical interfaces (parallel to the $\left[\begin{array}{lll}0 & 0 & 1\end{array}\right]$ direction). Accordingly, the normal plastic strain along the [0 01$]$ direction is accumulated by the horizontal interfacial motion, but not by the vertical interfacial dislocation motion along the [001] direction. The strain accumulating and non-accumulating periods associated with these processes can be clearly distinguished in the top diagram of Fig. 2(a) (solid black line).

The vertical interfacial dislocation motion is driven by the line tension force that acts to minimize the dislocation core energy. The line tension force is associated with the dislocation configuration trapped in the vertical channels,

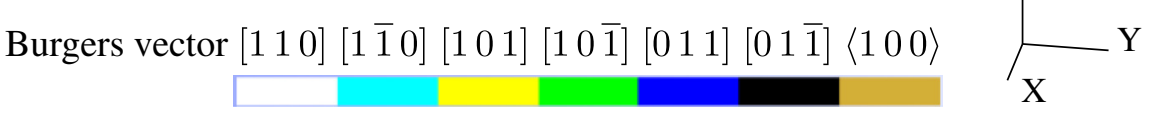

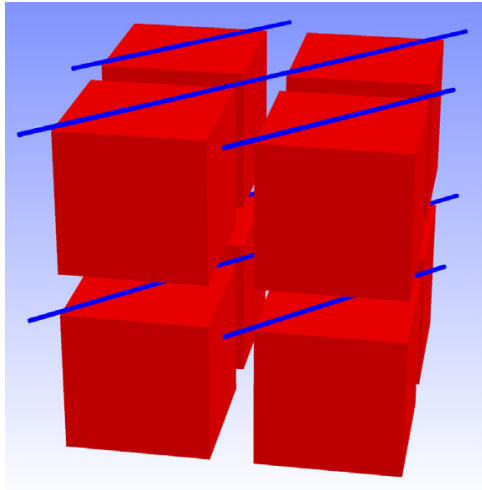

(a) mixed dislocations

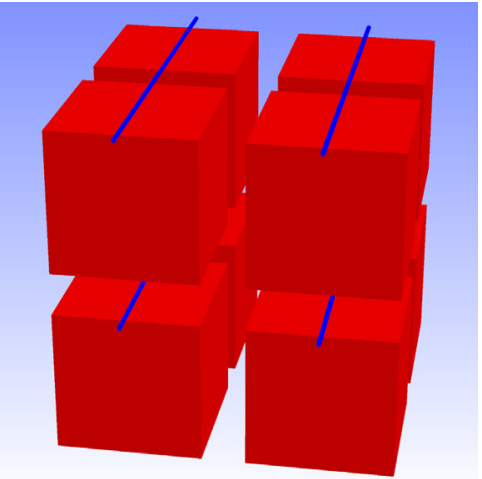

(b) edge dislocations

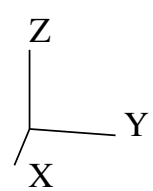

Fig. 1. Initial configurations of widely separated dislocations. 

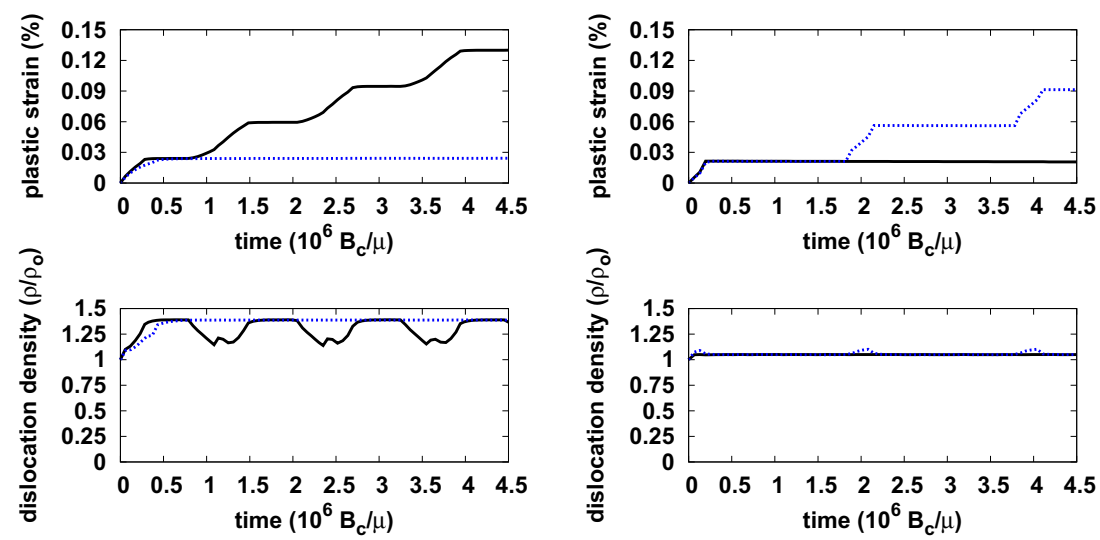

(a) mixed dislocations

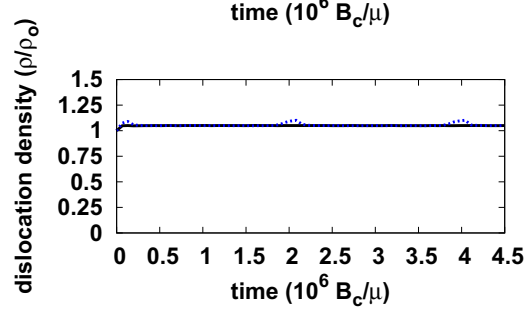

(b) edge dislocations

Fig. 2. Plastic strain accumulation and dislocation density evolution during the interfacial motion of widely separated dislocations: the simulation considering the misfit stresses is plotted with a dotted blue line $(.$.$) , and the simulation without considering the misfit stresses is plotted with a solid black$ line (-). (For interpretation of the references to color in this figure legend, the reader is referred to the web version of this article.)
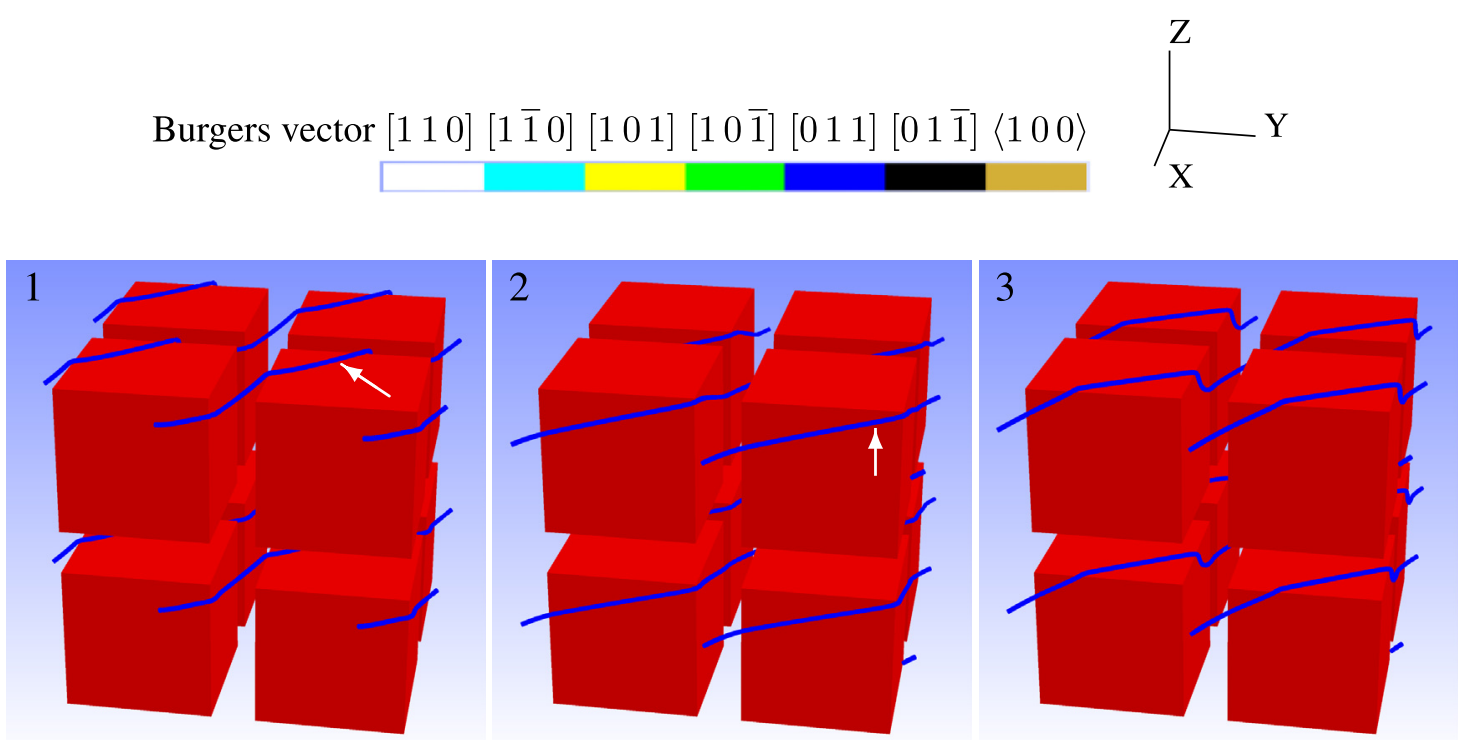

(a) without misfit stresses
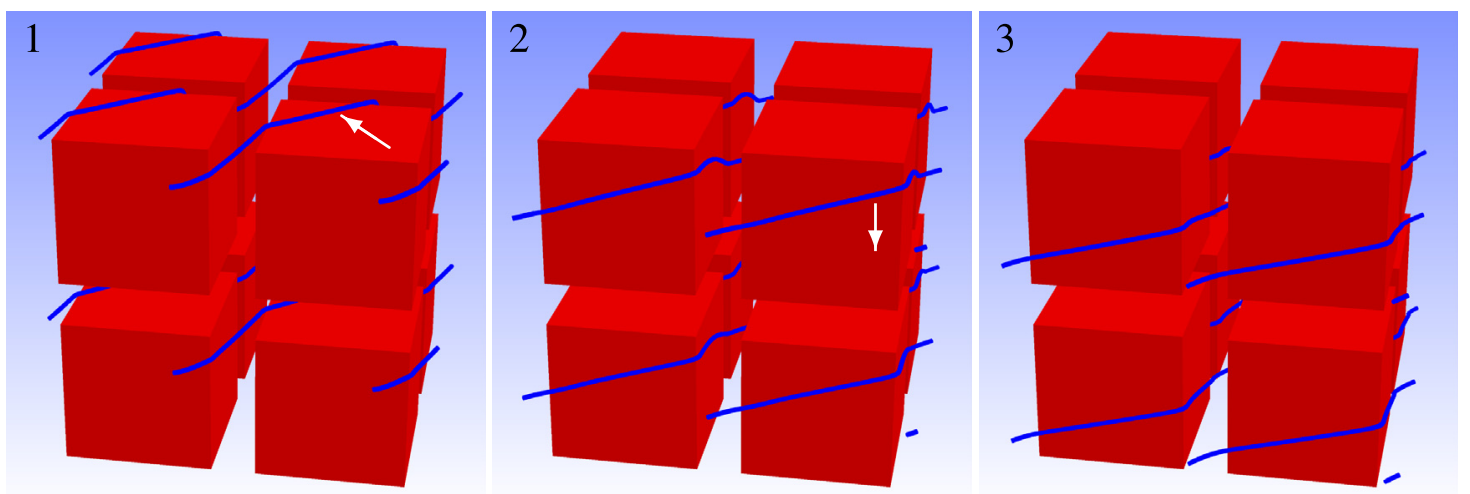

(b) with misfit stresses

Fig. 3. Interfacial motion of mixed dislocations. 
which gradually reduces as the dislocation moves out of the vertical channels and changes its line configuration. Such a process is reflected by the dislocation density change in the simulation shown in the bottom diagram of Fig. 2(a) (solid black line).

The simulation of mixed dislocations considering the misfit stresses is shown in Fig. 3(b). It can be seen that in the presence of the misfit stresses, the dislocations move downwards once they are trapped in the vertical channels (Fig. 3(b), images 2 and 3). Note that the simulated dislocations have a $1 / 2$ [0 111$]$ Burgers vector, which are not activated by the misfit stress component along the [100] direction. The misfit stress component along the [001] direction does not affect the vertical interfacial dislocation motion. Only the force exerted by the misfit stress component along the $[010]$ direction can overcome the line tension force, and drag the dislocation moving downwards along the vertical channels. This slow dislocation movement along the vertical channels does not contribute to the plastic strain along the [001] direction, and causes no change in the dislocation density (Fig. 2(a), dotted blue lines).

\subsubsection{Edge dislocations}

The simulation of edge dislocations without considering the misfit stresses is shown in Fig. 4(a). The dislocations primarily move along the horizontal interfaces towards the edges of the cuboidal $\gamma^{\prime}$ precipitates (Fig. 4(a), image 1), later glide across the vertical channels and reach the vertical interfaces of neighboring precipitates (Fig. 4(a), image 2 ), and lastly move downwards along the vertical interfaces (Fig. 4(a), image 3). The vertical dislocation motion along the [001] direction also results from the line tension force. Due to the different dislocation configurations, the line tension force for the trapped edge dislocation drags downwards along the vertical interfaces (Fig. 4(a), image 2), while the line tension force for the trapped mixed dislocation pulls it upwards along the vertical interfaces (Fig. 3(a), image 2). Since the trapped edge dislocation is mostly a straight line, the associated line tension force is low. The vertical dislocation motion is so slow that the dislocations have not reached the edges of the precipitates by the end of the simulation. This slow dislocation movement along the vertical interfaces does not contribute to the plastic strain along the $[001]$ direction, and causes no change in the dislocation density (Fig. 2(b), solid black lines).

The simulation of edge dislocations considering the misfit stresses is shown in Fig. 4(b). It can be seen that in the presence of the misfit stress, the dislocations have moved a long distance along the vertical interfaces to reach the edges of the precipitates (Fig. 4(b), images 2 and 3). The dislocations will then glide across the horizontal channels and move again on the horizontal interfaces, which is not shown in the paper. The cross-channel glide and horizontal interfacial dislocation motion contribute to the plastic strain along the [001] direction, which is reflected in the plastic strain-time diagram of Fig. 2(b) (dotted blue line).
3.2. Interfacial motion and interactions of closely spaced dislocations

The simulations with closelyspaced dislocation arrays are intended to reveal the effect of dislocation interactions on interfacial dislocation motion. For the eight activated slip systems under the [0 011$]$ loading, the dislocation interaction types between two slip systems are given in Table 1, where the interaction type abbreviations for the selected reference slip system pairs are in blue boldface. The initial interfacial dislocation networks are constructed by considering one or two activated $\langle 011\rangle\{11 \overline{1}\}$ slip systems in the horizontal channels that deposit mixed dislocations of opposite line senses on opposite interfaces (Fig. 5). Depending on the glide planes, the interfacial dislocation networks are either in the form of parallel dislocation arrays or in the form of square dislocation networks. Under low external stresses with dislocation climb enabled at high temperatures, dislocation arrays are easily relaxed to low-energy configurations (being regular) as frequently observed in creep experiments $[7,11,13,14,57,59]$. Perfect regularity is adopted in the initial configurations to avoid any heterogeneous dislocation distribution with high local back-stresses that can reverse the slip deposition in the beginning of the simulations. The average dislocation spacing in the interfacial dislocation networks is $90 \mathrm{~nm}$. Each group of the interfacial dislocation networks corresponds to a dislocation density of $5 \times 10^{13} \mathrm{~m}^{-2}$.

Fig. 6 shows the plastic strain accumulation and dislocation density evolution with time in these simulations, which are largely affected by the different types of interfacial dislocation interactions. For the simulations starting with the same initial configuration, the plastic strain accumulation and dislocation density evolution also varies when conducted with the misfit stresses (dotted blue line in Fig. 6) and and without the misfit stresses-(solid black line in Fig. 6).

In terms of the dislocation density evolution, the simulation results can be categorized into three groups. For the simulations of collinear or Lomer junction forming slip systems, the dislocation density starts to decrease at the onset of creep and can drop to zero at the end of the simulation (Fig. 6(a) and (b)). For the simulations of a single slip system or Hirth junction forming slip systems, a drop in dislocation density occurs during the intermediate stage before it remains constant until the end of the simulation (Fig. 6(c) and (d)). For simulations of coplanar or glissile junction forming slip systems, the dislocation density remains almost constant during the simulation (Fig. 6(e) and (f)).

\subsubsection{Collinear slip systems}

Fig. 7 shows the interfacial dislocation motion and interaction on collinear slip systems under external and misfit stresses. Comparing Figs. 5(a) and 7(a), the collinear annihilation of the two dislocation arrays results in an array of dislocations in zig-zag shapes. These dislocations 


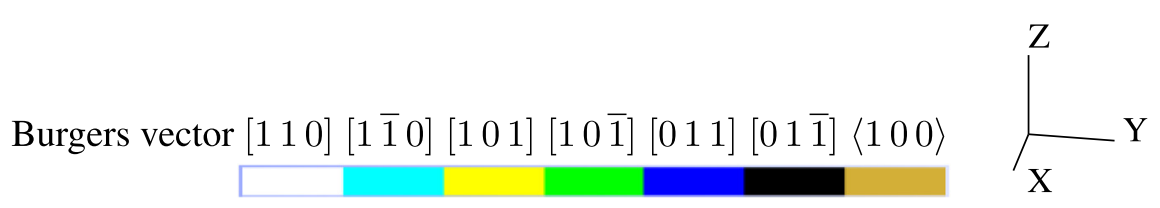
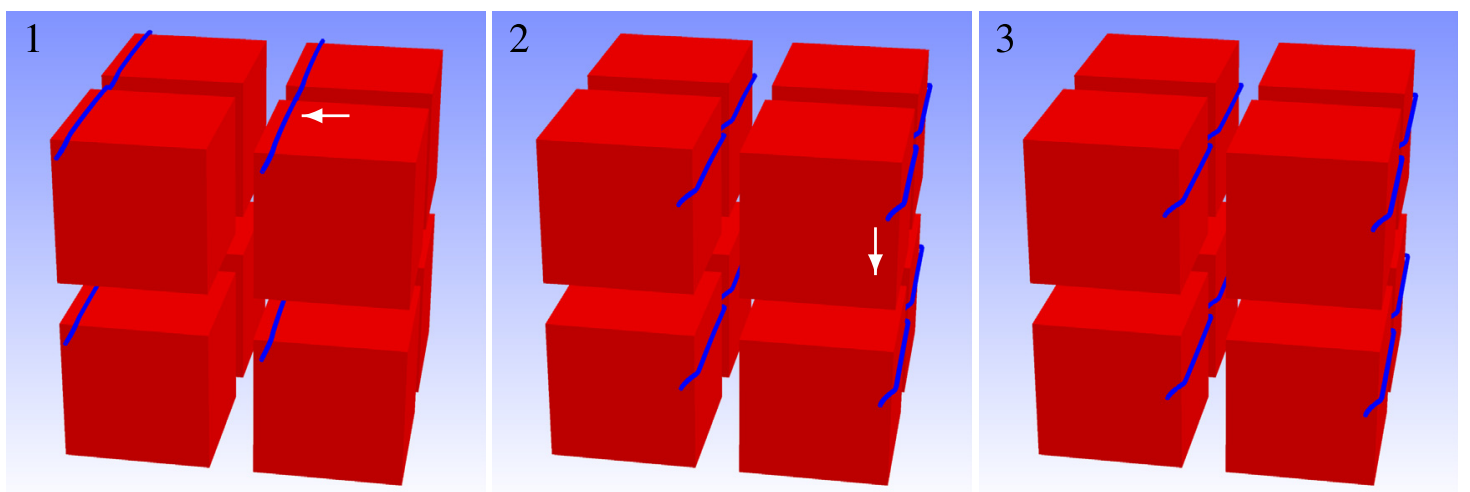

(a) without misfit stresses
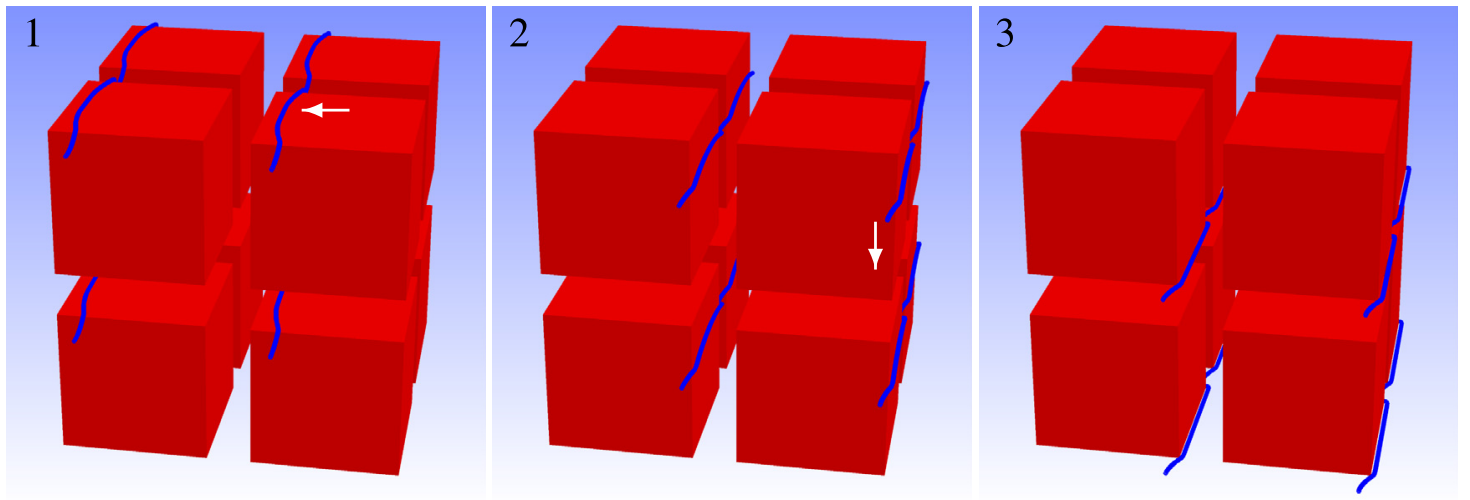

(b) with misfit stresses

Fig. 4. Interfacial motion of edge dislocations.

Table 1

Dislocation interaction types between two activated slip systems under the [001] tension: self $(\mathrm{S})$, collinear $(\mathrm{C})$, coplanar $(\mathrm{P})$, Lomer junction (L), glissile junction $(\mathrm{G})$ and Hirth junction $(\mathrm{H})$. The interaction type abbreviations for the selected reference slip system pairs are in blue boldface. Some Hirth junction interactions are unlikely to occur, and the corresponding abbreviations are colored gray. See more details in the discussion section.

\begin{tabular}{|c|c|c|c|c|c|c|c|c|c|}
\hline Slip system & No. & 1 & 2 & 3 & 4 & 5 & 6 & 7 & 8 \\
\hline$\left[\begin{array}{lll}0 & 1 & 1\end{array}\right]\left(\begin{array}{lll}1 & 1 & \overline{1}\end{array}\right)$ & 1 & $\mathbf{S}$ & & & & & & & \\
\hline$\left[\begin{array}{lll}0 & 1 & 1\end{array}\right]\left(\begin{array}{lll}1 & \overline{1} & 1\end{array}\right)$ & 2 & C & $\mathrm{S}$ & & & & & & \\
\hline$\left[\begin{array}{lll}0 & 1 & \overline{1}\end{array}\right]\left(\begin{array}{lll}\overline{1} & 1 & 1\end{array}\right)$ & 3 & $\mathbf{H}$ & $\mathrm{H}$ & $\mathrm{S}$ & & & & & \\
\hline$\left[\begin{array}{llll}0 & 1 & \overline{1}\end{array}\right]\left(\begin{array}{lll}1 & 1 & 1\end{array}\right)$ & 4 & $\mathrm{H}$ & $\mathrm{H}$ & $\mathrm{C}$ & $\mathrm{S}$ & & & & \\
\hline$\left[\begin{array}{lll}1 & 0 & 1\end{array}\right]\left(\begin{array}{lll}1 & 1 & \overline{1}\end{array}\right)$ & 5 & $\mathbf{P}$ & $\mathrm{G}$ & $\mathrm{G}$ & $\mathrm{L}$ & $\mathrm{S}$ & & & \\
\hline$\left[\begin{array}{lll}1 & 0 & 1\end{array}\right]\left(\begin{array}{lll}1 & 1 & 1\end{array}\right)$ & 6 & $\mathbf{G}$ & $\mathrm{L}$ & $\mathrm{P}$ & $\mathrm{G}$ & $\mathrm{C}$ & $\mathrm{S}$ & & \\
\hline$\left[\begin{array}{lll}1 & 0 & \overline{1}\end{array}\right]\left(\begin{array}{lll}1 & \overline{1} & 1\end{array}\right)$ & 7 & $\mathrm{G}$ & $\mathrm{P}$ & $\mathrm{L}$ & $\mathrm{G}$ & $\mathrm{H}$ & $\mathrm{H}$ & $\mathrm{S}$ & \\
\hline$\left[\begin{array}{lll}1 & 0 & \overline{1}\end{array}\right]\left(\begin{array}{lll}1 & 1 & 1\end{array}\right)$ & 8 & $\mathbf{L}$ & $\mathrm{G}$ & $\mathrm{G}$ & $\mathrm{P}$ & $\mathrm{H}$ & $\mathrm{H}$ & $\mathrm{C}$ & $\mathrm{S}$ \\
\hline
\end{tabular}

are later straightened due to their line tension (Fig. 7(b)), and become straight edge dislocations along the $\left[\begin{array}{ll}1 & 0\end{array}\right]$ direction (Fig. 7(c)). Such edge dislocations are very often observed in creep experiments [7,16,57] and are more effective at relieving the misfit stress than the glide-deposited mixed dislocations [13]. It is explicitly shown here that these edge dislocations are formed through the short-range reactions between the glide-deposited mixed dislocations on collinear slip systems.

Similar to the previous simulations, the edge dislocations can enter the vertical channels by glide, and move along the vertical interfaces in a combination of glide and climb. The edge dislocations now at the vertical interfaces have entered the vertical channels from opposite horizontal interfaces and thus have opposite line senses. The antiparallel edge dislocations are moving in opposite directions along the vertical channels (Fig. 7(c)). In the meantime, more edge dislocations are entering the vertical channels (Fig. 7(d)). The dislocations entering from the bottom interfaces are moving up along the vertical interfaces to 


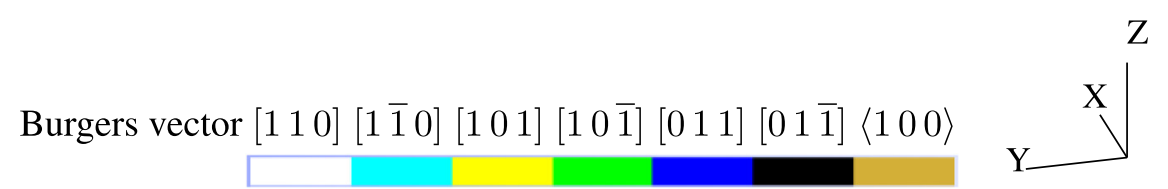

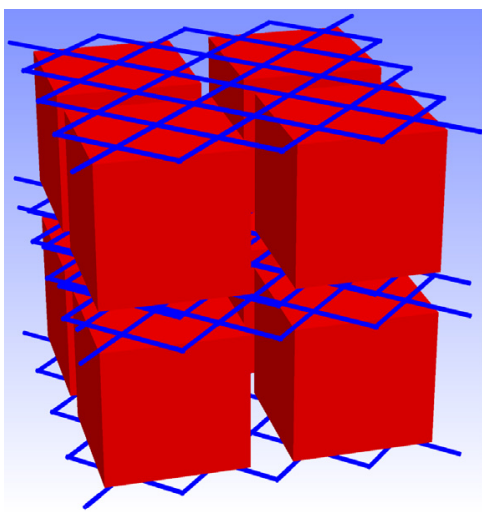

(a) collinear

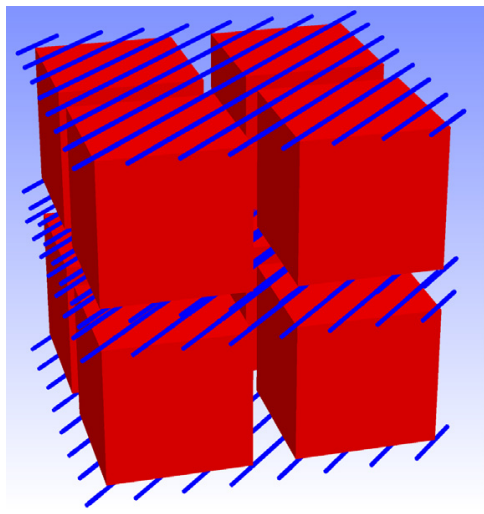

(c) self

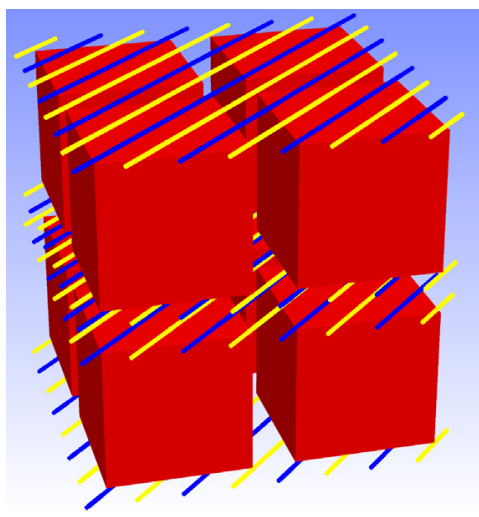

(e) coplanar

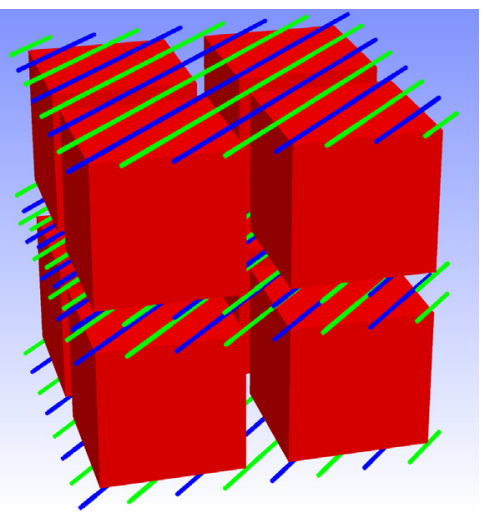

(b) Lomer

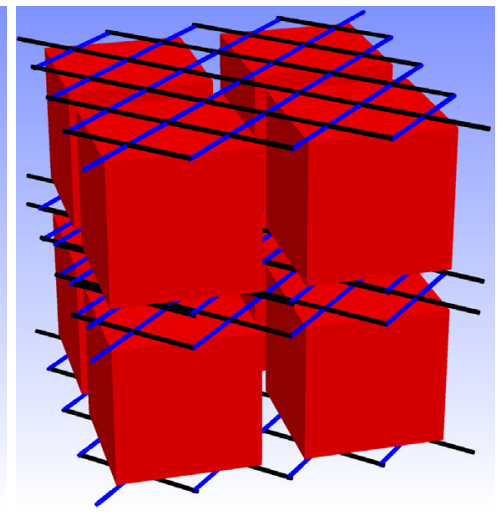

(d) Hirth

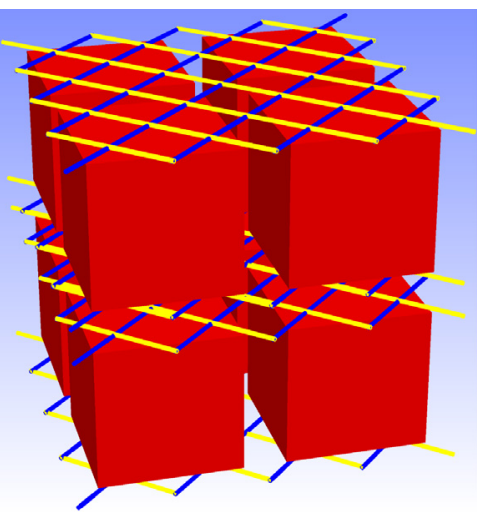

(f) glissile

Fig. 5. Initial configurations of interfacial dislocation networks involving different types of dislocation interactions.

meet the dislocations moving on the top interfaces at the precipitate corners (Fig. 7(e)). The antiparallel dislocations annihilate partially and form rectangular dislocation loops (Fig. 7(f)), which shrink at the edges of the precipitates (Fig. $7(\mathrm{~g})$ ), and eventually disappear (Fig. 7(h)).
The remaining dislocations are all moving along the interfaces in the vertical channels, where the misfit stress component along the [001] direction partially cancels the external stress. The mutual attraction of the antiparallel dislocations on opposite interfaces can overcome the 


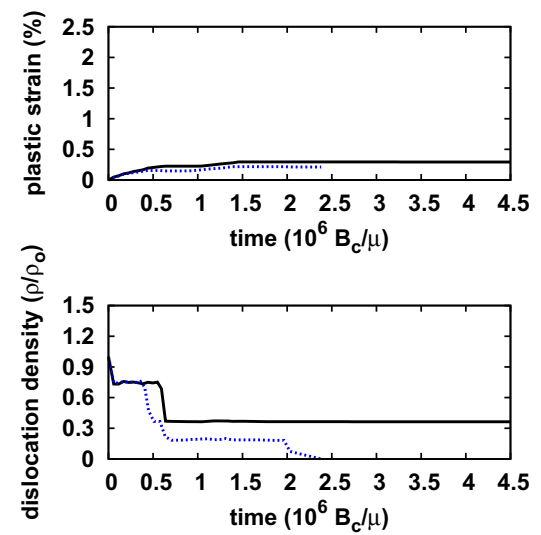

(a) collinear
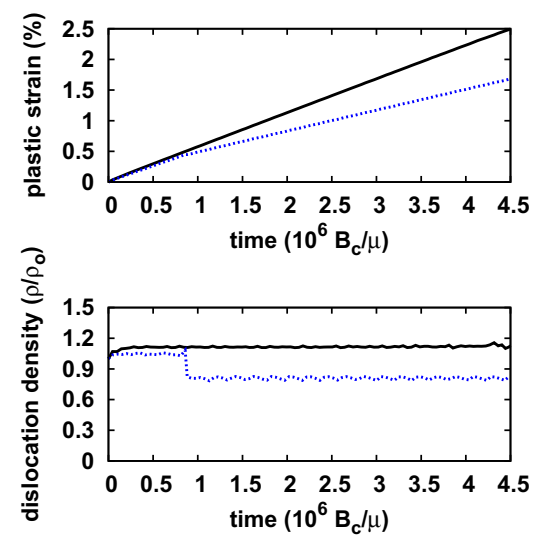

(c) self
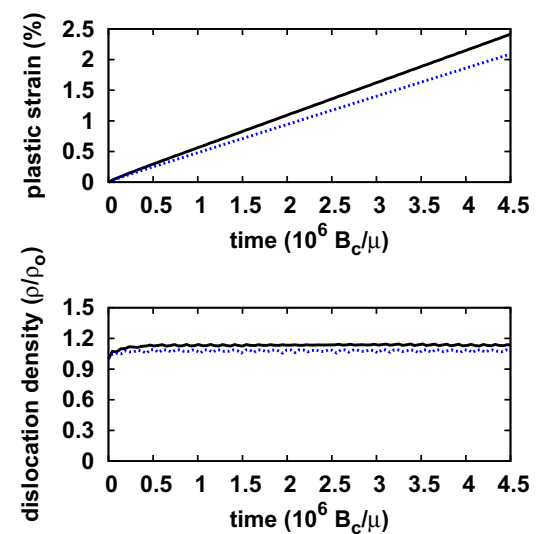

(e) coplanar

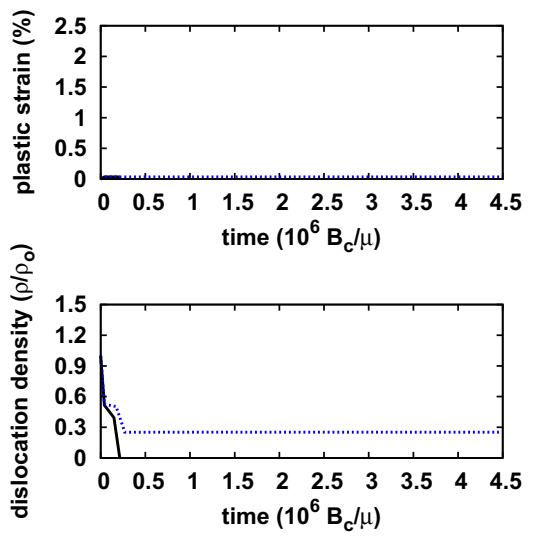

(b) Lomer
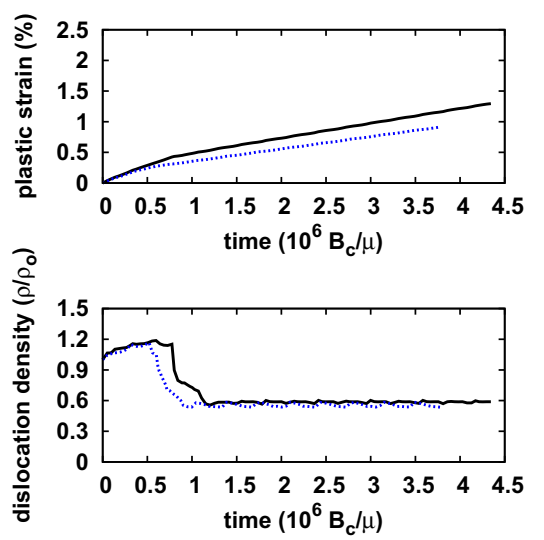

(d) Hirth
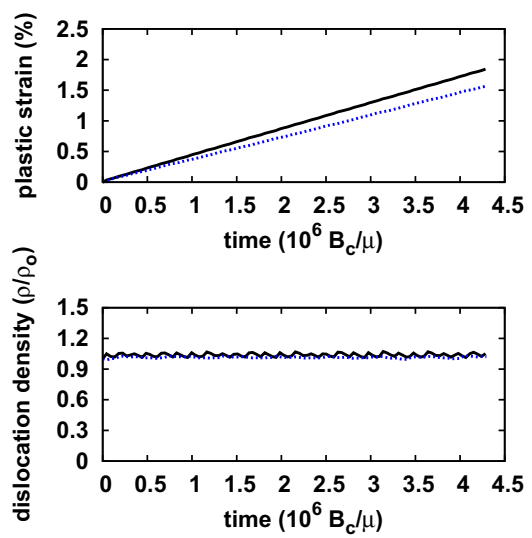

(f) glissile

Fig. 6. Plastic strain accumulation and dislocation density evolution during the interfacial motion of dislocation networks involving different types of dislocation interactions: the simulation considering the misfit stresses is plotted with a dotted blue line ( . .), and the simulation without considering the misfit stresses is plotted with a solid black line (-). (For interpretation of the references to color in this figure legend, the reader is referred to the web version of this article.)

remaining external stress, and leads to the annihilation of a pair of dislocations in the vertical channels (Fig. 7(h) and (i)). The mutual repulsion of dislocations of the same sign on the same interface pushes another pair of dislocations out of the vertical channels, which are then moving along the horizontal interfaces (Fig. 7(i)). The following snapshots of this simulation are not shown here. These dislocations will move to the opposite edges of the horizontal interfaces, re-enter the vertical channels and annihilate each other. The dislocation density drops to zero after this last annihilation, which can be seen in the dislocation density-time diagram in Fig. 6(a) (dotted blue line).

The simulation of the collinear interfacial dislocation motion and interactions in the absence of the misfit stresses is not shown here. The main difference is that the annihilation across the vertical channels in the presence of the misfit stress shown in Fig. 7(h) and (i) does not occur in the absence of the misfit stress. In the latter case, a constant dislocation 

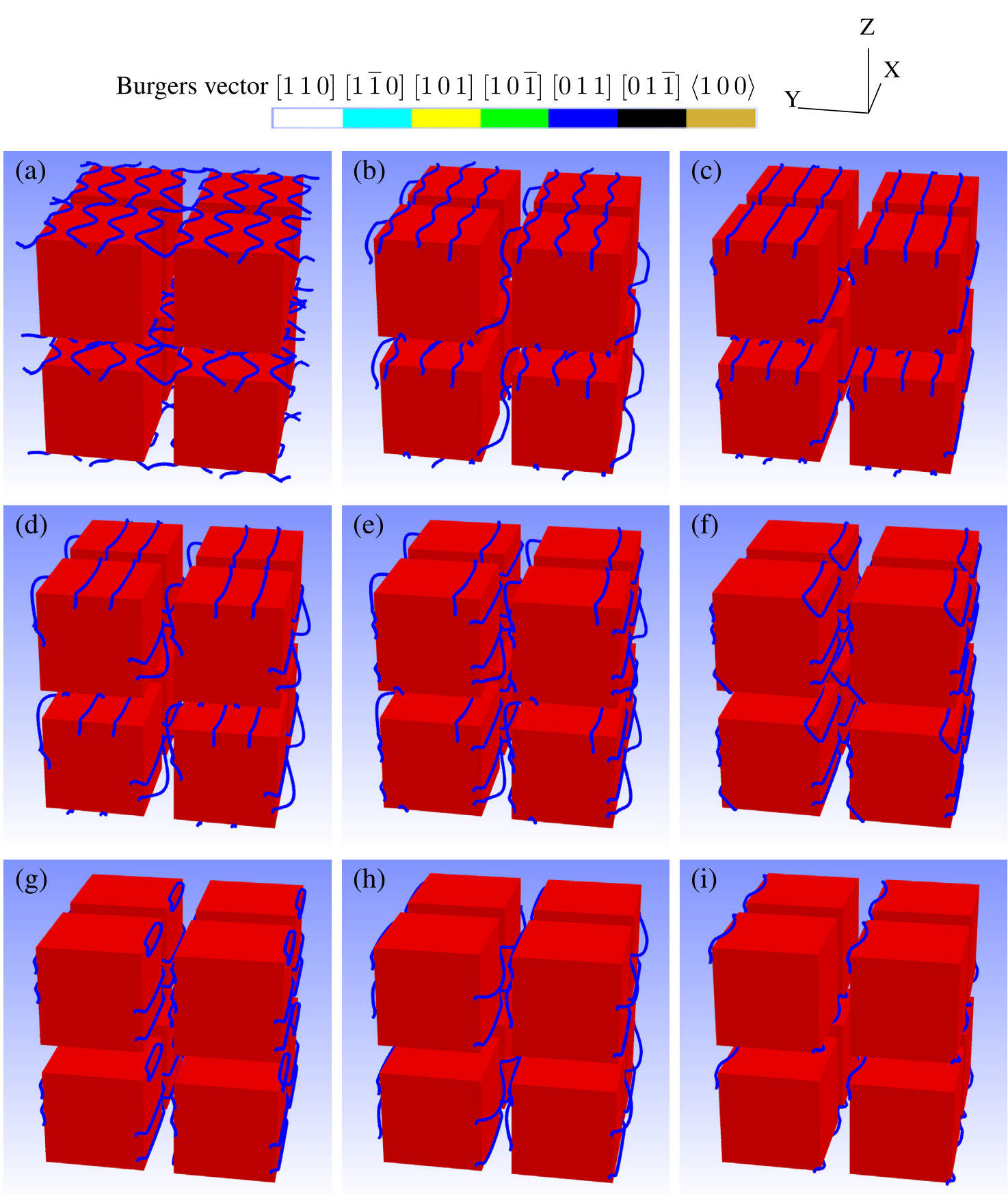

Fig. 7. Interfacial dislocation motion and interaction on collinear slip systems.

density is maintained until the end of the simulation, which is recorded in the dislocation density-time diagram in Fig. 6(a) (solid black line).

\subsubsection{Lomer junction forming slip systems}

Fig. 8 shows the interfacial dislocation motion and interaction on Lomer junction forming slip systems under the external stress. These two slip planes and the $\gamma / \gamma^{\prime}$ horizontal interfaces intersect along a common line direction, and the initial network consists of parallel dislocations (Fig. 5(b)). The dislocations are mainly moving along the horizontal interfaces by a combination of glide and climb, and are partially squeezed into the vertical channels by glide (Fig. 8(a)). The $1 / 2[011]$ and $1 / 2$ [10 $\overline{1}]$ dislocations attract each other and merge to $1 / 2$ [110] junctions first at the vertical channels, where they are closer to each other (Fig. 8(b)). The junction zipping completes as the parent $1 / 2\left[\begin{array}{lll}0 & 1 & 1\end{array}\right]$ and $1 / 2$ [ $\left.10 \overline{1}\right]$ dislocations approach each other on the horizontal interfaces (Fig. 8(c)). The newly formed 1/2 [110] dislocations are not activated by the external stress along the [001] direction. The dislocations of opposite signs on opposite interfaces move towards each other due to their mutual attraction (Fig. 8(d)). 

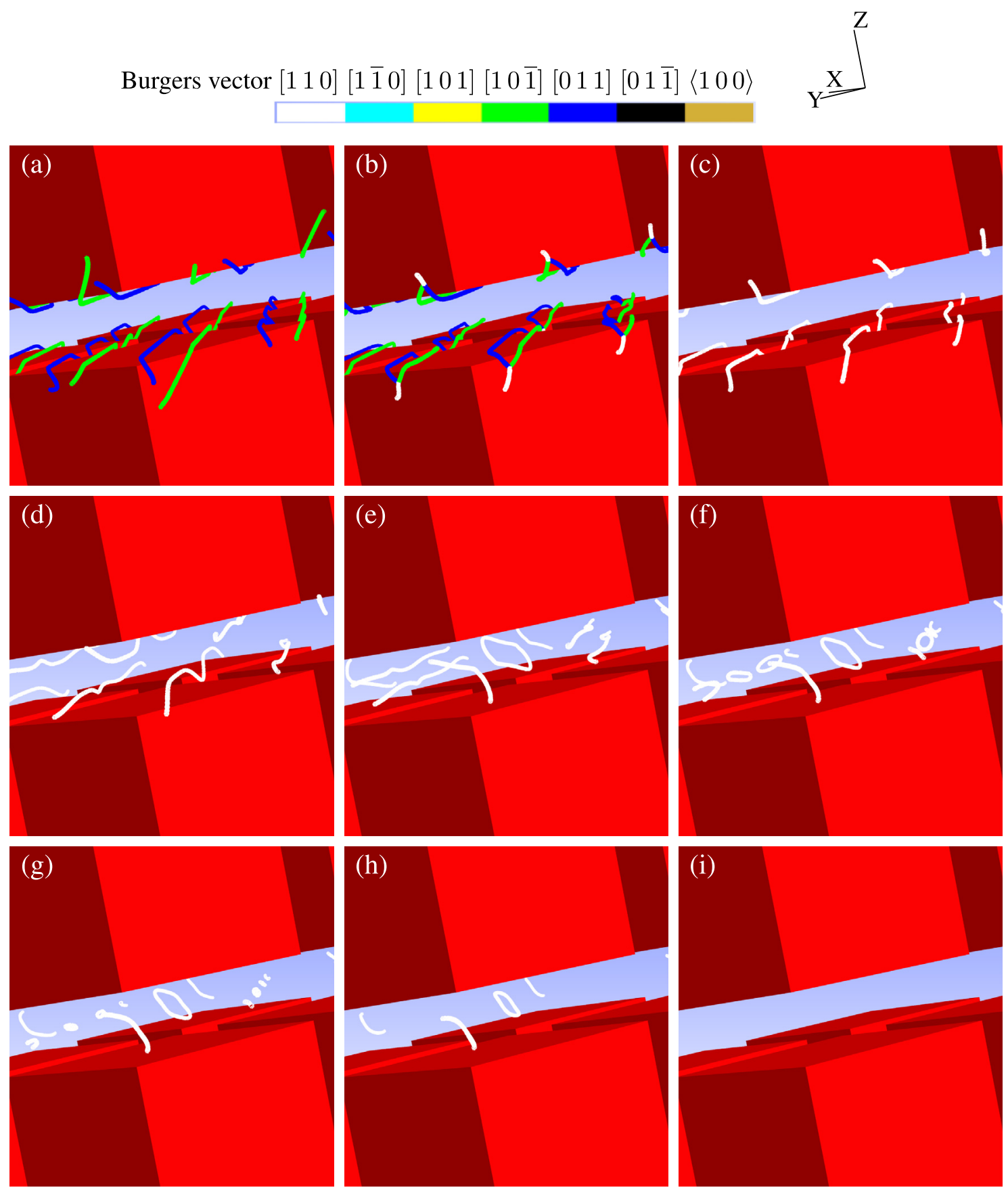

Fig. 8. Interfacial dislocation motion and interaction on Lomer junction forming slip systems.

The junction dislocations inherit the curved line configurations of their parent dislocations that were partially squeezed into the vertical channels. As the dipolar dislocations approach each other, some parts of the dislocations are directly annihilated, and the remaining parts of the dislocations form dislocation loops (Fig. 8(e) and (f)). Depending on the curvatures of the junction dislocations, the loops formed due to their partial annihilation are of different sizes. The break-up of dislocation dipoles into prismatic loops has been frequently observed in high-temperature deformation or subsequent annealing [60-63]. In our case, each loop formed is not entirely prismatic, but a mixture of glide and prismatic loops. The loops shrink by both glide and climb. As dislocations glide faster than they climb, the glide parts of the loops shrink first, which transforms the mixed loops into prismatic loops. Consequently, the loop shrinkage is accompanied by the rotation of the dislocation loop plane (Fig. 8(g) and (h)). Eventually all the dislocations are annihilated, as the loops self-annihilate by areal shrinking (Fig. 8(i)).

The simulation of the Lomer junction forming slip systems considering the misfit stresses is not shown. The main 

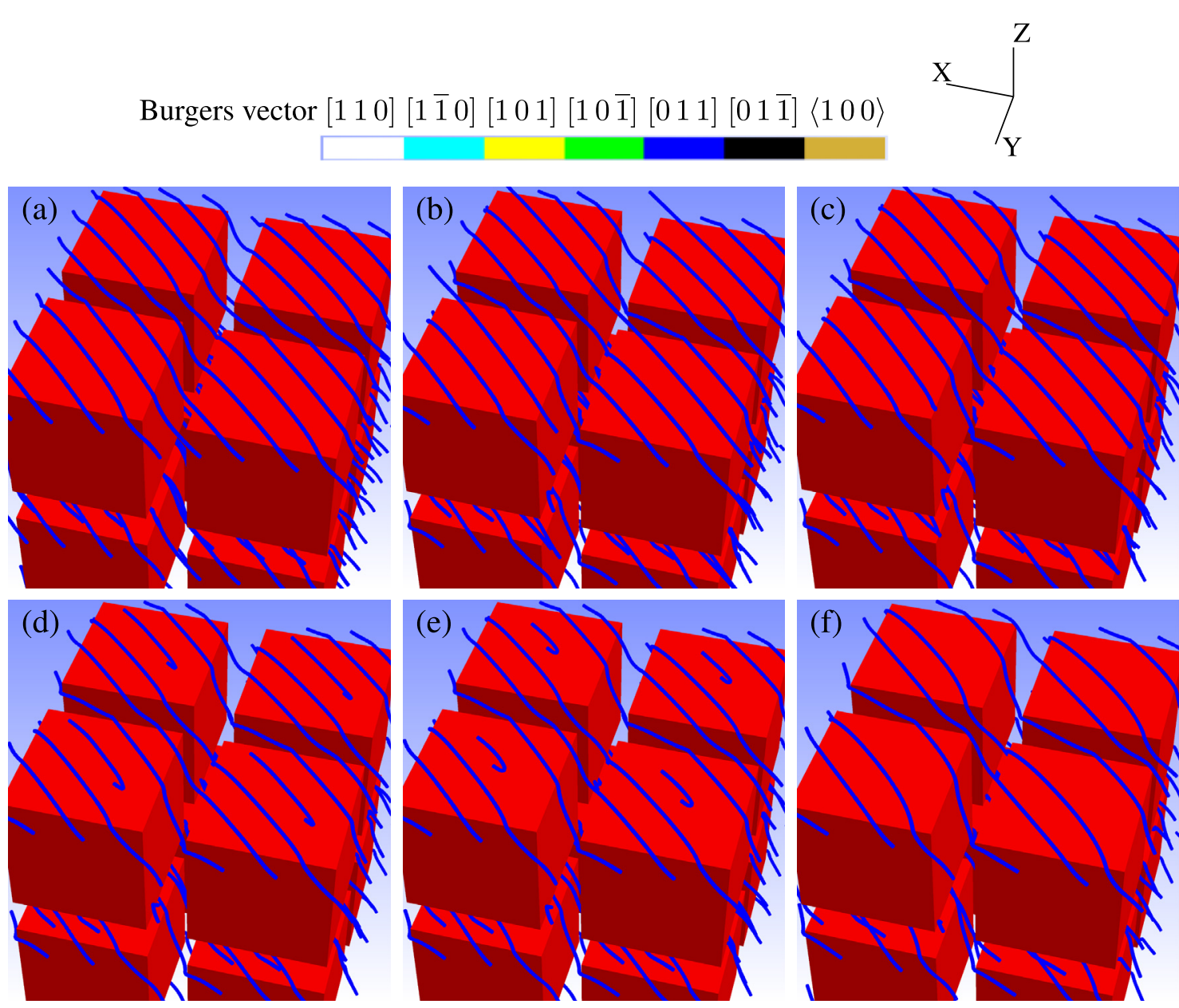

Fig. 9. Interfacial dislocation motion and self-interaction on a single slip system.

difference is that the annihilation of the $1 / 2[110]$ junction dislocations are partially prevented in the presence of the misfit stress. The remaining dipolar dislocations are far away from each other, and separated by the misfit stress on opposite horizontal interfaces. The dislocation density-time diagram in Fig. 6(b) records the partial annihilation in the presence of the misfit stress (dotted blue line) and the full annihilation in the absence of the misfit stress (solid black line).

\subsubsection{Single slip system}

Fig. 9 shows the interfacial dislocation motion and selfinteraction on a single slip system under the external and misfit stresses. The initial configuration consists of dipolar arrays of mixed dislocations on opposite horizontal interfaces (Fig. 5(c)). Probst-Hein et al. [5] have conducted static dislocation calculations of such dislocation networks to evaluate the Peach-Koehler (PK) forces on the network dislocations as a function of the number of dislocations in the network. As the number of network dislocations increases (the network dislocation spacing decreases), the dislocation internal stresses eventually overcome the external and misfit stresses, which causes the sign change of the PK forces. Our dislocation dynamics simulation shows that due to the trapping of dislocations at the vertical channels, the local dislocation spacing there becomes smaller than the average dislocation spacing (Fig. 9(a) and (b)). When the internal stresses exceed the external stress at the channel crosses after 172000 time steps, ${ }^{1}$ the dipolar dislocations there move towards each other and annihilate (Fig. 9(c)). The annihilation is similar to the Lomer junction annihilation, which consists of two general steps, i.e. partial annihilation leads first to loop formation (Fig. 9(d)), and these loops self-annihilate by areal shrinking (Fig. 9(e) and (f)). Due to the simulation view presented here, only halves of the dislocation loops can be seen. After this annihilation, the internal stresses are reduced and are no longer high enough to overcome the external stress. A constant dislocation density is then maintained until the end of the simulation (Fig. 6(c), dotted blue line in the dislocation density-time diagram).

The interfacial dislocation motion and self-interaction in the absence of the misfit stresses is not shown. As observed in Fig. 3, without the inward dragging caused by the misfit stress, the mixed dislocations can easily move out of the

\footnotetext{
The simulations in the paper generally run for one million time steps, unless terminated earlier due to a complete annihilation.
} 
vertical channels due to the outward pulling by the line tension force. The local dislocation spacing decrease and dipole annihilation in the presence of the misfit stress shown in Fig. $2(\mathrm{e})$ and (f) does not occur in the absence of the misfit stress. The dislocation network structure then hardly changes, and the dislocation density is almost constant during the simulation (Fig. 6(c), solid black line in the dislocation density-time diagram).

\subsubsection{Hirth junction forming slip systems}

Fig. 10 shows the interfacial dislocation motion and interaction on Hirth junction forming slip systems under external and misfit stresses. The initial configuration consists of dipolar square dislocation networks on opposite horizontal interfaces (Fig. 5(d)). The $60^{\circ} 1 / 2$ [0 111$]$ and $1 / 2\left[\begin{array}{lll}0 & 1 & \overline{1}\end{array}\right]$ dislocations in the same network are repulsive to each other, and do not form Hirth junctions (Fig. 10(a)). As these dislocations move to the vertical channels, their mutual repulsion pushes the $1 / 2\left[\begin{array}{lll}0 & 1 & \overline{1}\end{array}\right]$ dislocations out of the vertical channels, and the $1 / 2$ [011] towards the center of the vertical channels (Fig. 10(b) and (c)). The dislocations that entered the vertical channels from the bottom interfaces are moving up along the vertical interfaces to meet the dislocations moving on the top

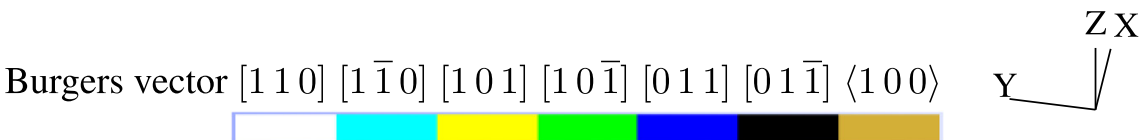
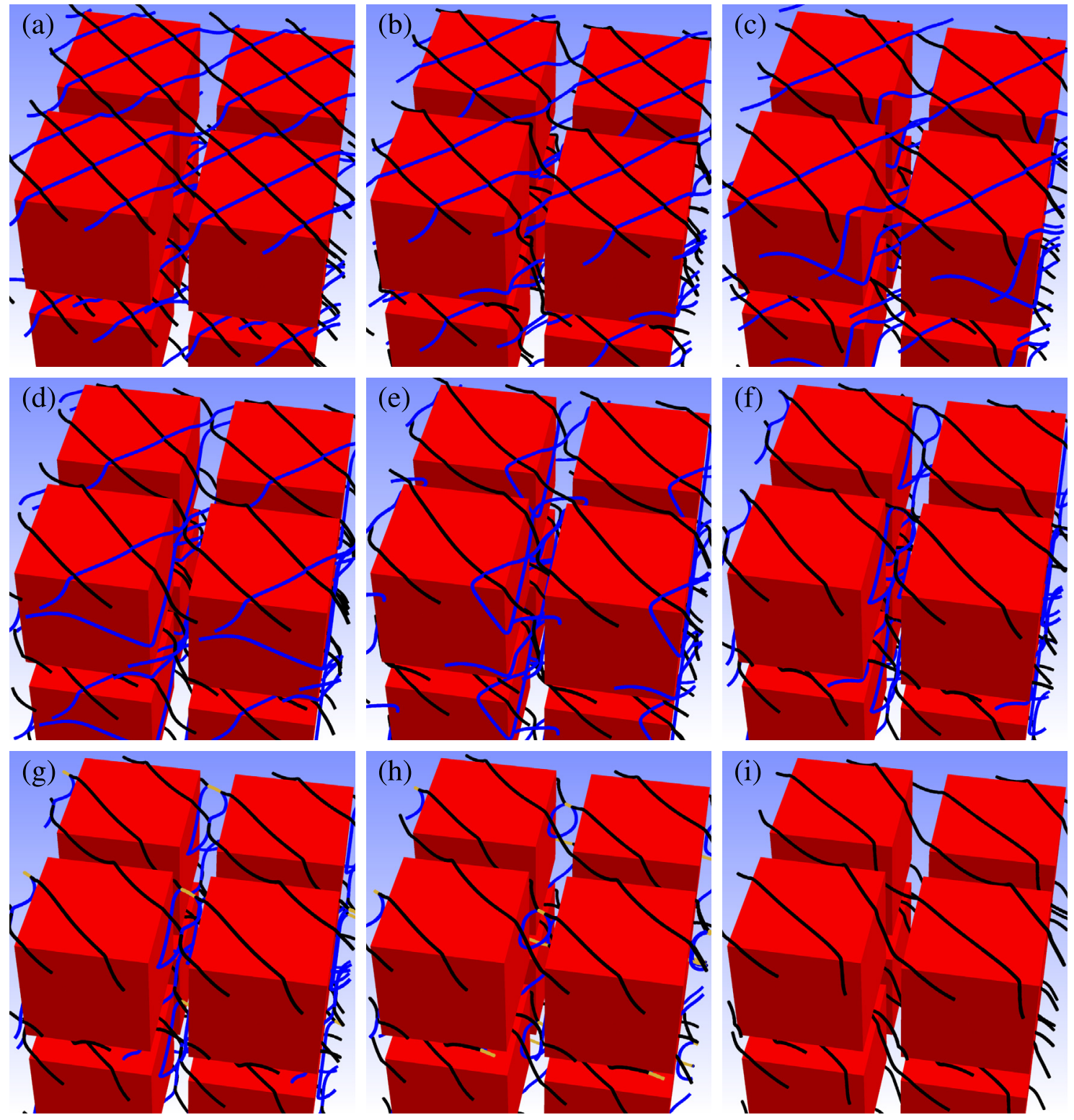

Fig. 10. Interfacial dislocation motion and interaction on Hirth junction forming slip systems. 
interfaces at the precipitate corners (Fig. 10(d)). Portions of the dislocations are annihilated in the vertical channels normal to the $\left[\begin{array}{lll}0 & 1 & 0\end{array}\right]$ direction, which leaves irregular dislocation loops at the precipitate corners and in the vertical channels normal to the $\left[\begin{array}{lll}0 & 1 & 0\end{array}\right]$ direction (Fig. 10(e)). The triangular parts of the dislocation loops shrink and disappear first at the precipitate corners, and the remaining debris of the dislocation loops continue to shrink in the vertical channels normal to the [010] direction (Fig. 10(f)). In the meantime, the $1 / 2\left[\begin{array}{lll}0 & 1 & \overline{1}\end{array}\right]$ dislocations enter the vertical channels, and partially merge with the shrinking $1 / 2$ [0 111$]$ dislocation loops to form [0 10$]$ Hirth junctions (Fig. $10(\mathrm{~g})$ ). As the 1/2 [0 1 1] dislocation loops shrink to disappear, the [010] Hirth junctions are later unzipped (Fig. 10(h) and (i)).

The simulation of the Hirth junction forming slip system without considering the misfit stresses is not shown. In the absence of the misfit stress, the mutual repulsion of $1 / 2$ $\left[\begin{array}{lll}0 & 1 & 1\end{array}\right]$ and $1 / 2\left[\begin{array}{lll}0 & 1 & \overline{1}\end{array}\right]$ dislocations still leads to the separation of the dislocation networks and the complete annihilation of the $1 / 2$ [ $\left.\begin{array}{lll}0 & 1 & 1\end{array}\right]$ dislocations. However, the movement of the $1 / 2$ [0 111$]$ dislocations along the vertical channels becomes slow, and the corner encounter of antiparallel dislocations in the presence of the misfit stress shown in Fig. 10(d) and (e) does not occur in the absence of the misfit stress. The annihilation starts at the channel crosses, which forms dislocation loops that shrink to disappear in the horizontal and vertical channels. The delayed annihilation due to the absence of the misfit stress can be seen in the dislocation density-time diagram of Fig. 6(d)

\subsubsection{Coplanar slip systems}

The interfacial dislocation motion and interaction on coplanar slip systems is shown in Fig. 11(a) for the simulation in the absence of the misfit stresses, and in Fig. 11(b) for the simulation in the presence of the misfit stresses. The initial configuration consists of dipolar arrays of parallel dislocations on opposite horizontal interfaces (Fig. 5(e)), similar to the Lomer junction and self-interaction cases (Fig. 5(b) and (c)). During the whole simulations, the coplanar

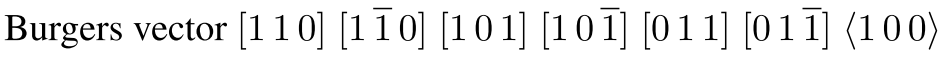

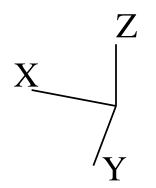

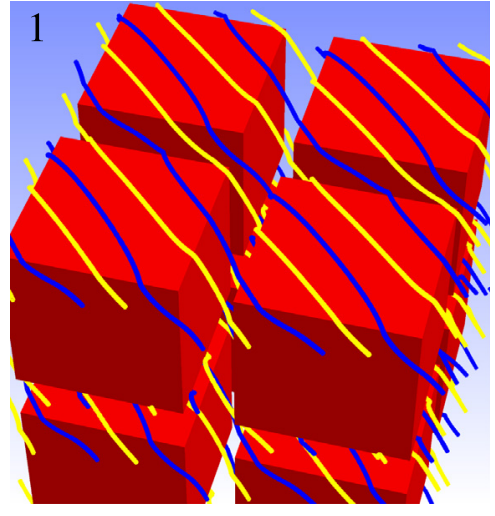

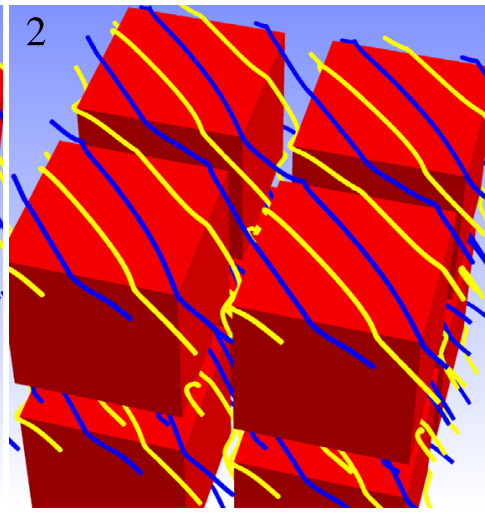

(a) without misfit stress
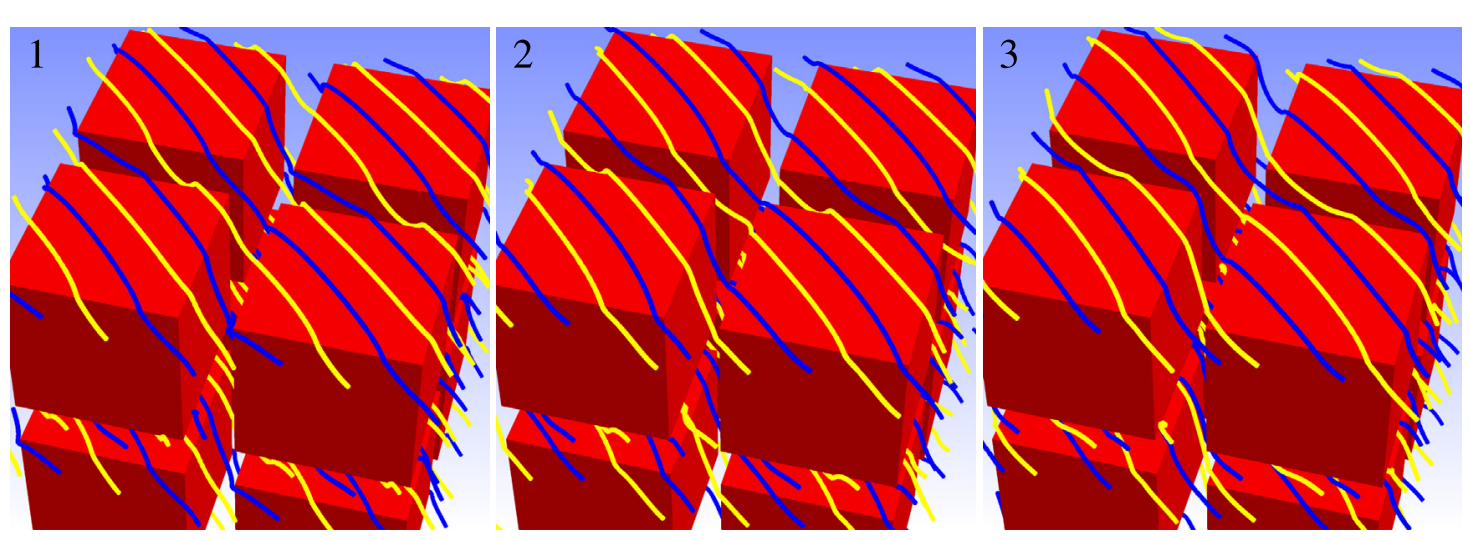

(b) with misfit stress

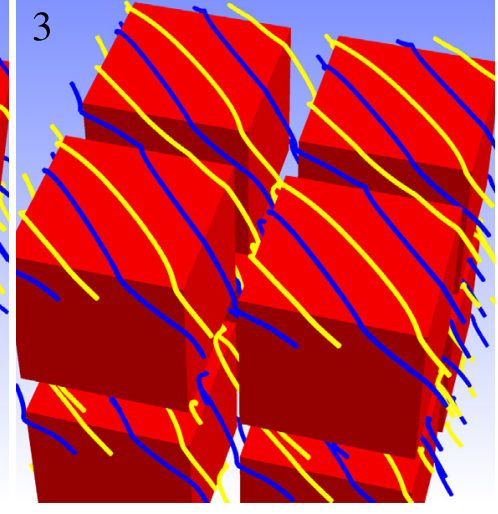

Fig. 11. Interfacial dislocation motion and interaction on coplanar slip systems. 
649 interfacial dislocations move through the $\gamma / \gamma^{\prime}$ structures in a 650 steady state (Fig. 11). Unlike the Lomer junction case, the 651 mixed dislocations of different Burgers vectors on the copla652 nar slip systems are repulsive to each other, and do not merge 653 to form dislocation junctions. Unlike the self-interaction 654 case, dislocation annihilation does not occur on the coplanar 655 slip systems even in the presence of the misfit stress. The dis656 location trapping at the vertical interfaces due to the misfit 657 stress can be seen by comparing Figs. 11(a) and (b). The 1/ $6582\left[\begin{array}{lll}0 & 1 & 1\end{array}\right]$ dislocations are trapped in the vertical channels nor-

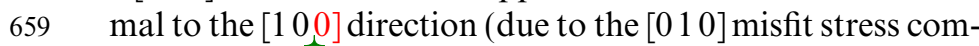
660 ponent), and $1 / 2[101]$ dislocations are trapped in the 661 vertical channels normal to the [0 10$]$ direction (due to the $662[100]$ misfit stress component). The dislocation density is 663 almost constant during both simulations, but the strain rate 664 is lower in the presence of the misfit stress (Fig. 6(e)).

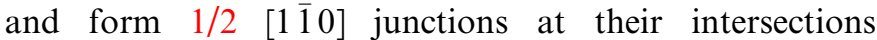
(Fig. 12(a)). As the junction zipping continues, the square dislocation networks are transformed into hexagonal dislocation networks (Fig. 12(b)).

The 1/2 [ $\left.1 \begin{array}{lll}1 & 1 & 0\end{array}\right]$ junction dislocations are formed approximately along the $[100]$ direction, and are not activated by the normal stress components along the [001] and [100] directions. The junction dislocations are driven by the misfit stress component along the [0 10$]$ direction to move along the $[001]$ direction, but do not move in the horizontal (001) plane. The horizontal motion of the hexagonal dislocation networks proceeds via the interfacial dislocation motion of the $1 / 2$ [0 111$]$ and $1 / 2$ [1 011$]$ parent dislocations, and involves junction zipping by the leading parent dislocations and junction unzipping by the tailing parent dislocations. The junction lengths increase when the tailing parent dislocations are trapped at the vertical interfaces, and decrease when the leading parent dislocations are trapped at the vertical interfaces (Fig. 12(c)). As the parent dislocations move out of the vertical channels, the junction dislocations tend to resume their original length (Fig. 12(d)). As more network dislocations pass through the vertical channels, the mesh of the hexagonal dislocation networks become irregular (Fig. 12(e) and (f)).
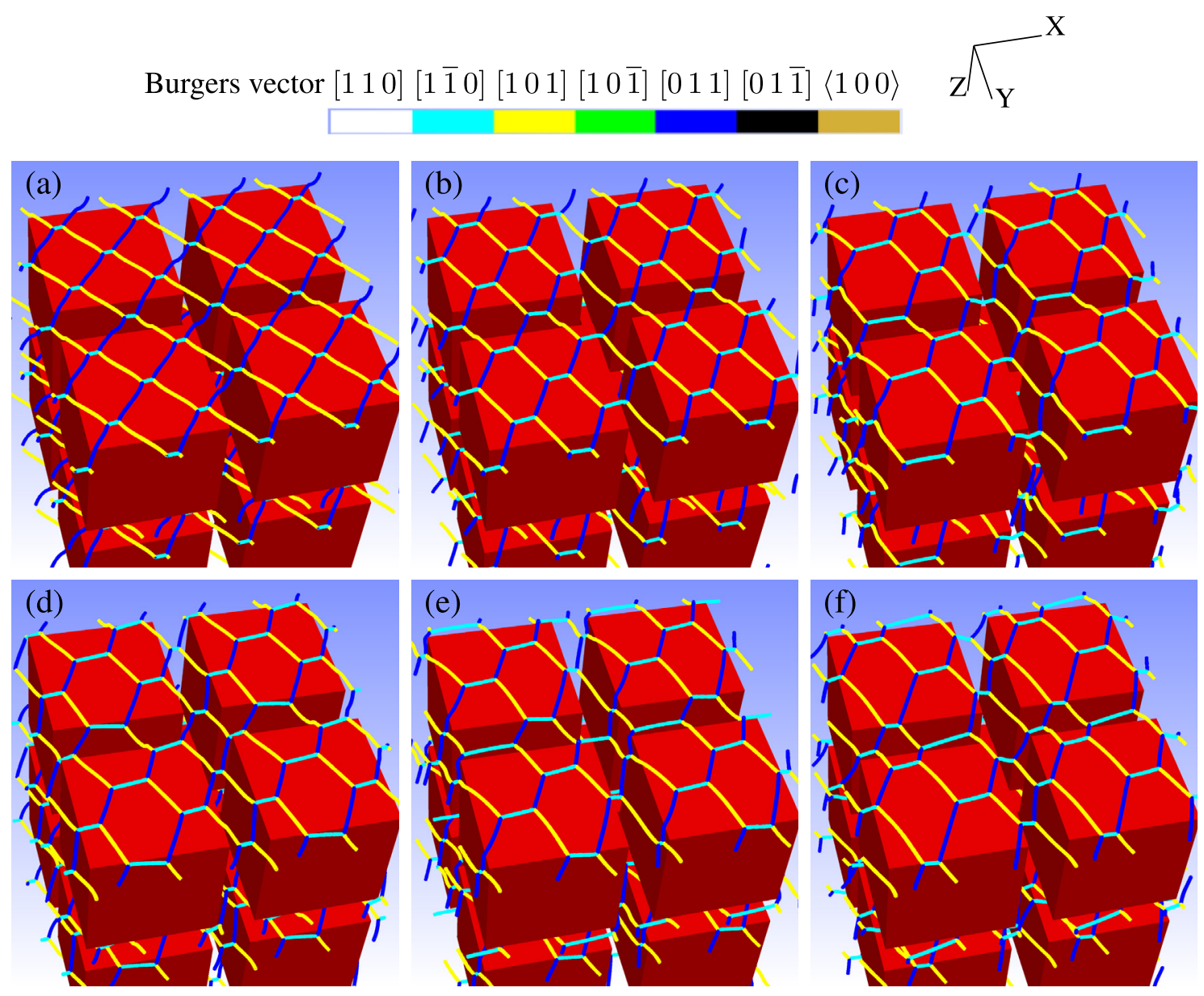

Fig. 12. Interfacial dislocation motion and interaction on glissile junction forming slip systems. 
The simulation of the glissile junction forming slip system without considering the misfit stresses is not shown. The main difference is that the mesh of the hexagonal dislocation networks becomes less irregular in the absence of the misfit stress than those in the presence of the misfit stress as shown in Fig. 12(e) and (f). The dislocation density is almost constant during both simulations, but the strain rate is lower in the presence of the misfit stress (Fig. 6(f)).

\section{Discussion}

During high-temperature low-stress creep of single-crystal superalloys, the tensile stress along the [00 1$]$ direction would ideally activate eight $\langle 011\rangle\left\{\begin{array}{lll}1 & 1 & 1\end{array}\right\}$ slip systems simultaneously, but in reality the slip systems are gradually activated in a random sequence $[10,11,13-15,57]$. Due to the available sources in the sample or slight deviations of the sample alignment with the loading axis, only one or two slip systems are activated at the early stage. The back-stresses associated with the interfacial dislocation networks prevent or slow down the activation of new slip systems under the applied low stress. Only after a recovery of the initial dislocation networks can other slip systems be substantially activated.

The simulation results show that interfacial dislocation motion and dynamic recovery in single-crystal superalloys can be strongly altered by interfacial dislocation interactions on the activated slip systems. Collinear annihilation transforms the square dislocation networks into parallel arrays of edge dislocations, which move freely along the horizontal and vertical interfaces, and annihilate each other at the precipitate corners. Lomer junction formation leaves long inactivated dislocation dipoles on opposite horizontal interfaces, which break up into dislocation loops that self-annihilate by areal shrinking. The interfacial dislocation motion on coplanar and glissile junction forming slip systems proceeds steadily, and does not lead to dynamic recovery at all. Interfacial dislocation motion and self-interaction on a single slip system develops high local stress due to the misfit stress trapping at the vertical interfaces, which causes partial annihilation of the interfacial dislocation networks starting at the channel crosses. The mutual repulsion between $60^{\circ}$ mixed dislocations on Hirth junction forming slip systems leads to the separation of the interfacial dislocation networks and annihilation of dislocations on one slip system in the vertical channels.

For self, collinear, coplanar, Lomer junction and glissile junction interactions, the simulation results of the selected reference slip system pairs are entirely equivalent to other activated slip system pairs involving the same type of dislocation interactions (see Table 1), which results from the 4fold rotation symmetry about the $[001]$ axis or the mirror symmetry with respect to $(100)$ or $(010)$ planes. For an example, the Lomer junction interactions between slip systems No. 1 and 8 can be transformed to the equivalent interactions between slip systems No. 6 and 2 through a $90^{\circ}$ rotation about the $[001]$ axis, and the glissile junction interactions between slip systems No. 1 and 6 can be transformed to the equivalent interactions between slip systems No. 2 and 8 through a $(100)$ plane reflection. For Hirth junction interactions, there are two non-equivalent variants due to the lost 4-fold rotation symmetry about the [1 00$]$ axis, which is caused by the favorable slip deposition on the horizontal interfaces. The other Hirth junction interaction variant has an initial configuration of parallel dislocation arrays similar to the Lomer junction and coplanar cases, but the interfacial dislocations of the two slip systems are repulsive and move in opposite directions on the horizontal interfaces. The dislocation structures become unstable in the beginning of the simulation, which are unlikely to appear in a double slip situation. These Hirth junction interaction variants are colored gray in Table 1.

Although these simulations of interfacial dislocation motion and interactions are essential to understand plastic deformation during high-temperature low-stress creep, the plastic strain-time curves obtained from these simulations should not be directly interpreted as creep curves. Several processes that could contribute or affect the creep strain accumulation are not considered in the model, which include the regeneration of interfacial dislocation networks after recovery, directional coarsening (rafting) of the $\gamma^{\prime}$ precipitates, and $\langle 100\rangle$ dislocations cutting of the rafted $\gamma^{\prime}$ precipitates. We shall discuss the potential effects of these processes on high-temperature low-stress creep based on the present simulation results.

The recovery of the interfacial dislocation networks relieves the internal back-stress, and the grown-in dislocations could again glide in the horizontal channels and form new interfacial dislocation networks. The softening effect of dynamic recovery is evident, as the glide of the grown-in dislocations proceeds much faster than the interfacial dislocation motion in a combination of glide and climb. Depending on the activated slip system, dynamic recovery and creep softening may initialize rapidly (collinear and Lomer junction forming slip systems) or not occur at all (coplanar and glissile junction forming slip systems), which can lead a strong scatter of creep curves at the early stage of high temperature low stress creep.

The dynamic recovery on the single slip system and Hirth junction forming slip systems does not occur initially but at a plastic strain up to $0.5 \%$. This is also the strain regime where substantial rafting takes place, reshaping the originally cuboidal morphology of the $\gamma^{\prime}$ precipitates into a lamellar-type arrangement perpendicular to the $\left[\begin{array}{lll}0 & 0 & 1\end{array}\right]$ loading axis $[6,12,15,56,59]$. Once the rafting has profoundly changed the shape of the precipitates into such a lamellar morphology, dynamic recovery associated with the vertical interfacial motion will no longer occur. The hardening effect of rafting shall be expected due to the prevention of further dynamic recovery in the vertical channels.

Dynamic recovery and creep deformation in the rafted $\gamma / \gamma^{\prime}$ microstructures are mainly related to $\langle 100\rangle$ dislocations cutting the $\gamma^{\prime}$ precipitates [12]. Several mechanisms on the $\langle 100\rangle$ dislocation formation in single-crystal 
810 superalloys have been proposed in the literature $811[11,12,57,58,64]$. One of the mechanisms is that the $\langle 100\rangle$ dislocations are formed as Hirth junctions [11,12,57]. Our simulation result shows that the Hirth junctions are not formed directly by the glide-deposited $60^{\circ}$ dislocations, but at the later stage of high-temperature low-stress creep when the glide-deposited dislocations have gone through a large line-orientation change during the interfacial dislocation motion and interactions.

\section{Summary}

We simulated interfacial motion of mixed dislocations, edge dislocations and different types of dislocation networks in the channel structures of single-crystal superalloys.

For widely separated dislocations, the horizontal interfacial dislocation motion is mainly driven by the external stress, and the vertical interfacial dislocation motion is largely affected by the presence of the misfit stress. The line tension force, depending on the trapped dislocation configuration, can drive mixed and edge dislocations to move in opposite directions along the vertical interfaces.

For dipolar dislocation networks, the interfacial dislocation motion and dynamic recovery is dominated by interfacial dislocation interactions. The short-range reactions of collinear annihilation and Lomer junction formation lead to recovery at the early stage of high-temperature lowstress creep. The misfit stress induces and accelerates the dynamic recovery of the single slip system and Hirth junction forming slip systems, but slows down the steady interfacial dislocation motion on the coplanar and glissile junction forming slip systems.

It is found that $1 / 2\langle 011\rangle$ edge dislocations along the $\langle 100\rangle$ directions are formed by glide-deposited mixed dislocations on collinear slip systems. Such a finding provides a theoretical basis for creep models built on such dislocation configurations, but at the same time suggests the inability of these models to describe interfacial dislocation motion on slip systems involving other types of dislocation interactions.

The simulation of Hirth junction forming slip systems supports the theoretical assumption that the $\langle 100\rangle$ dislocations are formed as Hirth junctions. However, the Hirth junctions are only formed when the glide-deposited dislocations have gone through a large line-orientation change during the interfacial dislocation motion and interactions, which indicates that the $\langle 100\rangle$ dislocation formation and associated recovery process of $\langle 100\rangle$ dislocation cutting does not occur at the early stage of high-temperature lowstress creep.

\section{Acknowledgments}

We thank Ricardo Lebensohn for his continuous support and Michael Demkowicz for helpful discussions. This work was performed under the auspices of the US
Department of Energy by Lawrence Livermore National Laboratory under Contract DE-AC52-07NA27344.

\section{References}

[1] Rae CMF, Reed RC. Acta Mater 2007;55(3):1067-81.

[2] Vorontsov VA, Shen C, Wang Y, Dye D, Rae CMF. Acta Mater 2010;58(12):4110-9.

[3] Pollock TM, Argon AS. Acta Metall Mater 1992;40(1):1-30.

[4] Vattre A, Devincre B, Roos A. Acta Mater 2010;58(6):1938-51.

[5] Probst-Hein M, Dlouhy A, Eggeler G. Acta Mater 1999;47(8):2497-510.

[6] Matan N, Cox DC, Rae CMF, Reed RC. Acta Mater 1999;47(7):2031-45.

[7] Zhang JX, Wang JC, Harada H, Koizumi Y. Acta Mater 2005;53(17):4623-33.

[8] Carry C, Strudel JL. Acta Metall 1977;25(7):767-77.

[9] Svoboda J, Lukas P. Acta Mater 1997;45(1):125-35.

[10] Epishin A, Link T. Philos Mag 2004;84(19):1979-2000.

[11] Link T, Epishin A, Klaus M, Bruckner U, Reznicek A. Mater Sci Eng A 2005;405(1-2):254-65.

[12] Sarosi PM, Srinivasan R, Eggeler GF, Nathal MV, Mills MJ. Acta Mater 2007;55(7):2509-18.

[13] Field RD, Pollock TM, Murphy WH. In: Antolovich SD, Stusrud RW, Mackay RA, Anton DL, Khan T, Kissinger RD, et al., editors. Superalloys 1992. Warrendale, PA: The Minerals, Metals and Materials Society; 1992. p. 557-66.

[14] Tian SG, Zhou HH, Zhang JH, Yang HC, Xu YB, Hu ZQ. Mater Sci Eng A 2000;279(1-2):160-5.

[15] Jacome LA, Noertershaeuser P, Heyer JK, Lahni A, Frenzel J, Dlouhy A, et al. Acta Mater 2013;61(8):2926-43.

[16] Carry C, Dermarkar S, StrudeL JL, Wonsiewicz BC. Metall Trans A 1979;10(7):855-60.

[17] Dlouhy A, Probst-Hein M, Eggeler G. Mater Sci Eng A 2001;309(SI):278-82.

[18] Carry C, StrudeL JL. Acta Metall 1978;26(5):859-70.

[19] Zhu Z, Basoalto H, Warnken N, Reed RC. Acta Mater 2012;60(12):4888-900.

[20] Kubin LP, Canova G, Condat M, Devincre B, Pontikis V, Brechet Y. Solid State Phenom 1992;23-24:455-72.

[21] Zbib HM, Rhee M, Hirth JP. Int J Mech Sci 1998;40(2-3):113-27.

[22] Schwarz KWJ. Appl Phys 1999;85(1):108-19.

[23] Ghoniem NM, Tong SH, Sun LZ. Phys Rev B 2000;61(2):913-27.

[24] Weygand D, Friedman LH, Van der Giessen E, Needleman A. Model Simul Mater Sci Eng 2002;10(4):437-68.

[25] Arsenlis A, Cai W, Tang M, Rhee M, Oppelstrup T, Hommes G, et al. Model Simul Mater Sci Eng 2007;15(6):553-95.

[26] Madec R, Devincre B, Kubin LP, Hoc T, Rodney D. Science 2003;301(5641):1879-82.

[27] Bulatov VV, Hsiung LL, Tang M, Arsenlis A, Bartelt MC, Cai W, et al. Nature 2006;440(7088):1174-8.

[28] Wu CC, Chung PW, Aubry S, Munday LB, Arsenlis A. Acta Mater 2013;61(9):3422-31.

[29] Akasheh F, Zbib HM, Hirth JP, Hoagland RG, Misra AJ. Appl Phys 2007;101(8).

[30] Rao SI, Dimiduk DM, Parthasarathy TA, Uchic MD, Tang M, Woodward C. Acta Mater 2008;56(13):3245-59.

[31] Tang H, Schwarz KW, Espinosa HD. Phys Rev Lett 2008;100(18).

[32] Motz C, Weygand D, Senger J, Gumbsch P. Acta Mater 2009;57(6):1744-54.

[33] Liu B, Raabe D, Eisenlohr P, Roters F, Arsenlis A, Hommes G. Acta Mater 2011;59(19):7125-34.

[34] Liu B, Eisenlohr P, Roters F, Raabe D. Acta Mater 2012 ;60(13-14):5380-90.

[35] Caillard D, Martin JL. Thermally activated mechanisms in crystal plasticity. Amsterdam: Pergamon Press; 2003.

[36] Friedel J. Dislocations. Oxford: Pergamon Press; 1964. 
[37] Hirth JP, Lothe J. Theory of dislocations. New York: Wiley; 1982.

[38] Mordehai D, Clouet E, Fivel M, Verdier M. Philos Mag 2008;88(6):899-925.

[39] Bako B, Clouet E, Dupuy LM, Bletry M. Philos Mag 2011;91(23):3173-91.

[40] Clouet E. Phys Rev B 2011;84(9).

[41] Davoudi KM, Nicola L, Vlassak JJJ. Appl Phys 2012;111(10).

[42] Keralavarma SM, Cagin T, Arsenlis A, Benzerga AA. Phys Rev Lett 2012;109(26).

[43] Danas K, Deshpande VS. Model Simul Mater Sci Eng 2013;21(4).

[44] Xiang Y, Srolovitz DJ. Philos Mag 2006;86(25-26):3937-57.

[45] Quek SS, Xiang Y, Srolovitz DJ. Acta Mater 2011;59(14):5398-410.

[46] Documentation of ParaDiS v2.5.1.

[47] Raabe D. Philos Mag A 1998;77(3):751-9.

[48] Mohles V. Mater Sci Eng A 2001;309(SI):265-9.

[49] Rao SI, Parthasarathy TA, Dimidukz DM, Hazzlediney PM. Philos Mag 2004;84(30):3195-215.

[50] Yashiro K, Kurose F, Nakashima Y, Kubo K, Tomita Y, Zbib HM. Int J Plast 2006;22(4):713-23.

[51] Vattre A, Devincre B, Roos A. Intermetallics 2009;17(12):988-94.
[52] Huang M, Zhao L, Tong J. Int J Plast 2012;28(1):141-58.

[53] Glatzel U, Feller-Kniepmeier M. Scr Metall 1989;23(11):1839-44.

[54] Ganghoffer JF, Hazotte A, Denis S, Simon A. Scr Metall Mater 1991;25(11):2491-6.

[55] Muller L, Glatzel U, Feller-Kniepmeier M. Acta Metall Mater 1992;40(6):1321-7.

[56] Pollock TM, Argon AS. Acta Metall Mater 1994;42(6):1859-74.

[57] Pollock TM, Field RD. In: Nabarro FRN, Duesbery MS, editors. Dislocations in solids, vol. 11. Amsterdam: North Holland; 2002. p. 547-618.

[58] Srinivasan R, Eggeler G, Mills M. Acta Mater 2000;48(20):4867-78.

[59] Reed RC, Matan N, Cox DC, Rist MA, Rae CMF. Acta Mater 1999;47(12):3367-81.

[60] Groves GW, Kelly AJ. Appl Phys 1962;33(1):456-60.

[61] Phillips D, Pletka B, Heuer A, Mitchell T. Acta Metall 1982;30(2):491-8.

[62] Lagerlof K, Mitchell T, Heuer A. Acta Metall 1989;37(12):3315-25.

[63] Gao Y, Zhuang Z, Liu Z, You X, Zhao X, Zhang Z. Int J Plast 2011;27(7):1055-71.

[64] Eggeler G, Dlouhy A. Acta Mater 1997;45(10):4251-62. 